\title{
Delay Performance of Some Scheduling Strategies in an Input Queuing ATM Switch with Multiclass Bursty Traffic
}

\author{
Lillykutty Jacob and Anurag Kumar, Senior Member, IEEE
}

\begin{abstract}
We consider an $N \times N$ nonblocking, space division, input queuing asynchronous transfer mode (ATM) cell switch, and a class of Markovian models for cell arrivals on each of its inputs. The traffic at each input comprises geometrically distributed bursts of cells, each burst destined for a particular output. The inputs differ in the burstiness of the offered traffic, with burstiness being characterized in terms of the average burst length. We analyze burst delays in the situation in which some inputs receive traficic with low burstiness and others receive traffic with higher burstiness. Three policies for head-ofthe-line contention resolution are studied: two static priority policies [viz., shorter-expected-burst-length-first (SEBF), longerexpected-burst-length-first (LEBF)] and random selection (RS).

Direct queuing analysis is used to obtain approximations for asymptotic (as $N \rightarrow \infty$ ) high and low priority mean burst delays with the priority policies. Simulation is used for obtaining mean burst delays for finite $N$ and for the random selection policy. $\mathrm{Nu}$ merical results show that, as the traffic burstiness increases, the asymptotic analysis can serve as a good approximation only for large switch sizes. Qualitative performance comparisons based on the asymptotic analysis are, however, found to continue to hold for finite switch sizes. It is found that the SEBF policy yields the best delay performance over a wide range of loads, while RS lies in between. SEBF drastically reduces the delay of the less bursty traffic (e.g., distributed computing trafic) while only slightly increasing the delay of the more bursty traficic, e.g., variable bit rate (VBR) video. LEBF causes severe degradation in the delay of less bursty traffic, while only marginally improving the delays of the more bursty trafic. $R S$ can be an adequate compromise if there is no prior knowledge of input traffic burstiness.
\end{abstract}

\section{INTRODUCTION}

$\mathbf{I}$ $\mathrm{N}$ this paper, we consider an $N \times N$ nonblocking, space division, input queuing asynchronous transfer mode (ATM) cell switch, schematically shown in Fig. 1. Performance analysis of such a switch has been done with various traffic assumptions. The studies in [5], [6], and [10] are based on a Bernoulli model for cell arrivals, with each cell independently

Manuscript received January 31, 1994; revised March 20, 1995. Approved by IEEE/ACM TRANSACtions on NeTwORKING Editor Hideo Miyahara. This work was supported in part by the Department of Electronics (Govt. of India), through its Education and Research Network (ERNET) Project at the Department of Electrical Communication Eng., Indian Institute of Science, Bangalore.

L. Jacob was with the Department of Electrical Communication Eng. Indian Institute of Science, Bangalore, 560 012, India. She is now with the Regional Engineering College, Calicut, Kerala, India (e-mail: lilly@reccal.ernet.in).

A. Kumar is with the Department of Electrical Communication Engineering, Indian Institute of Science, Bangalore 560 012, India (anurag@ece.iisc.ernet.in).

Publisher Item Identifier S 1063-6692(96)02717-3.

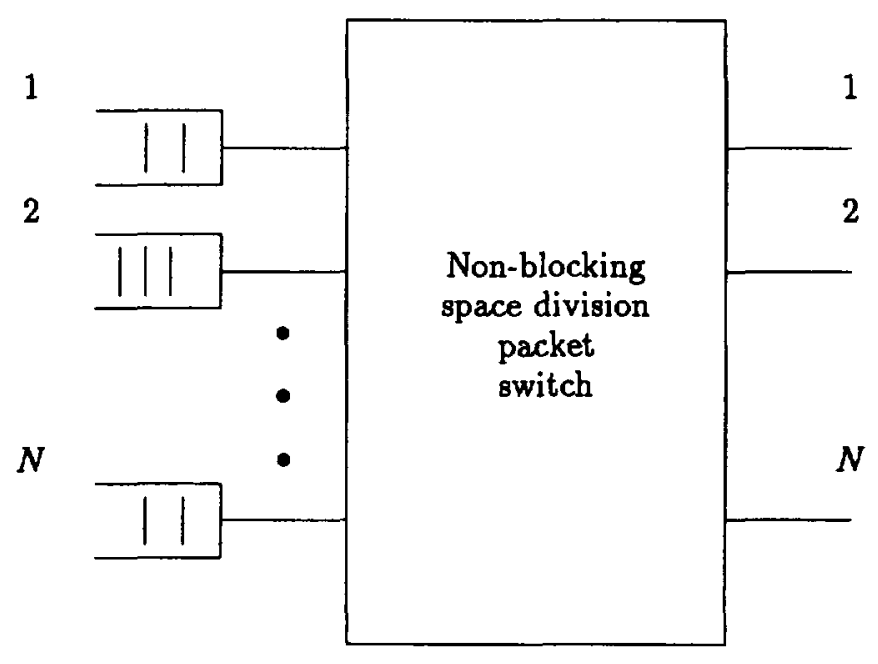

Fig. 1. $N \times N$ switch with input queuing.

requesting each output with equal probabililty. This is referred to in the literature as an independent and uniform traffic model. The switch performance under independent but nonuniform traffic (i.e., the routing probabilities of cells to outputs are unequal) was studied in [12]. Performance analysis with a certain model for correlated input traffic has been reported in [13].

While the study in [13] is concerned with correlated input traffic, it assumes that the traffic on each input link has the same statistical behavior. However, a "local access" ATM switch will receive traffic from metropolitan area networks (MAN's) and also directly from broadband integrated services digital networks (B-ISDN) terminals [1]. It can be expected that since MAN's aggregate traffic from relatively slow sources, the cells on ATM links emanating from MAN's will display low serial correlation in their demands for the output links. The traffic from a B-ISDN terminal, e.g., a high definition television (HDTV) source, however, can be expected to display high serial correlation, even to the extent of delivering consecutive cells destined for the same switch output. Motivated by this situation, we have considered the following input traffic model.

At each input, the cell arrival process is characterized by an $N+1$ state Markov chain $\left\{\xi_{n}, n \geq 1\right\}$ whose transition probabilities are depicted in Fig. 2. If $\xi_{n}=m, 1 \leq m \leq N$, then a cell destined for output $m$ arrives in the $n$th slot; 
whereas $\xi_{n}=\emptyset$ denotes that $n$th slot is empty. Clearly the number of consecutive slots with cells for the same output has a geometric distribution with mean $1 /(1-p)$. We refer to this batch of consecutive cells destined for the same output as a "burst" of cells for that output. Upon the completion of a burst to an output, with probability $q$ there is a geometrically distributed sequence of idle slots, and with probability $(1-q)$ a burst for a different output. The output for this new burst is chosen uniformly from among the other $(N-1)$ outputs. This cell arrival process model is similar to the one used in [3] and [14]. It follows that the cell arrival rate $\gamma$ (in cells per slot) is given as $\gamma=(1-q) /(1-p q)$. As discussed above, in certain situations, some input processes display low serial correlation and others high serial correlation. We model this by asserting that a fraction $\alpha$ of the $N$ inputs have traffic with mean burst length $1 /\left(1-p_{1}\right)$ (called type 1 inputs) and the rest of the $(1-\alpha) N$ inputs have traffic with mean burst length $1 /\left(1-p_{2}\right)$ (called type 2 inputs).

For input queuing with first-in first-out (FIFO) service discipline for each queue, a contention resolution scheme is needed in order to choose one head-of-the-line (HOL) cell when there are two or more input queues with their HOL cells destined for the same output. In most of the previous studies, it is assumed that among the $k \mathrm{HOL}$ cells contending for the same output, one cell is randomly (uniformly) selected for transmission across the switch fabric in one slot time. This is called the random selection (RS) policy. In [2] a priority selection policy is investigated. Each input receives two classes of traffic as independent Bernoulli streams. The priority introduced is mainly to support traffics with different delay requirements; e.g., real time traffic such as voice is assigned high priority to satisfy its strict delay requirement. We have shown [7], [8] that, when different input links have traffic with different statistical behavior, there can be a significant throughput advantage if, when the HOL cells from several input queues contend for the same output, the input with the smaller bursts (on the average) is given priority [referred to as shorter-expected-burst-length-first (SEBF)]. A remarkable finding is that, when there are two traffic classes, giving priority to less bursty traffic can give better throughput than if all the inputs were occupied by this less bursty traffic. Further, the results show that giving priority to the burstier traffic [referred to as longer-expected-burst-length-first (LEBF)] can result in a severe throughput degradation. We have done this by obtaining explicit expressions for the high and low priority saturation throughputs for the asymptotic case (the switch size $N \rightarrow x)$.

In this paper, we study the impact of the HOL contention resolution policies on the mean burst delay. Note that for traffic such as packet voice or video, burst delays are more meaningful than individual cell delays. In particular, we compare the RS, SEBF, and LEBF scheduling strategies by comparing the mean burst delays with these schemes when we have the multiclass bursty traffic scenario described above. In Section II, we perform a detailed delay analysis for $N$ (switch size) $\rightarrow \infty$ for the priority contention resolution scheme. Section II-A deals with the high priority mean burst delay, and Section II-B with the low priority mean burst delay.

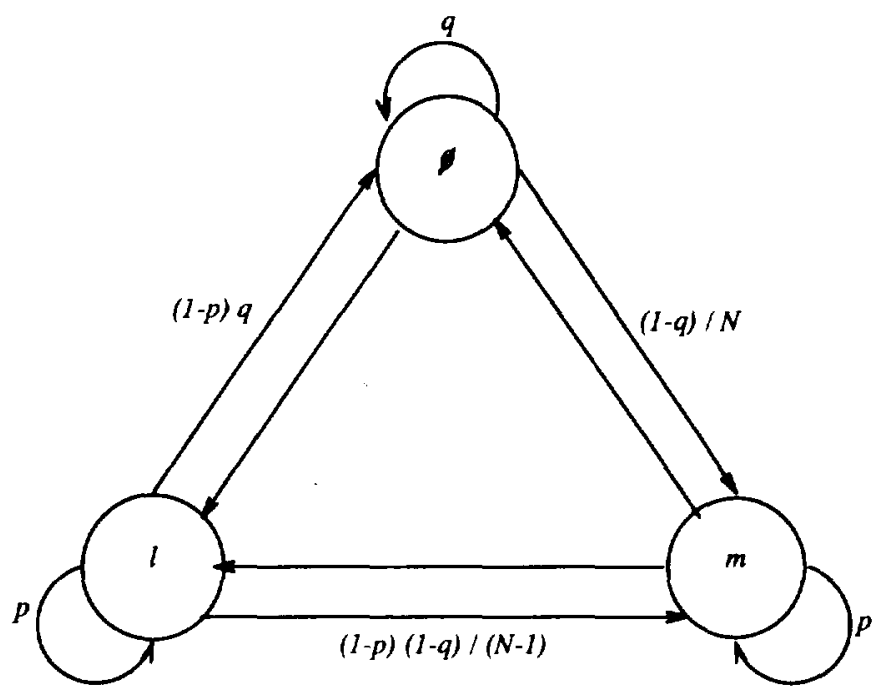

Fig. 2. Markov chain transition diagram for the cell arrival process at each input. There are $Y$ states in which the input is carrying cells destined for a particular output; only two such states $I$ and $m$ are shown.

Section III deals with the numerical results of the analysis of Section II and comparisons with simulation results for finite switch size $N$. For the random selection scheme the analysis with multiclass bursty traffic is intractable, and hence this is studied by simulation. A comparison of the three schemes (i.e.. RS, SEBF, and LEBF) based on the analytical and simulation results is presented in Section IV. We conclude in Section V.

\section{A. Notational Note}

We follow the convention that if $X$ denotes a continuous random variable, $X(x)$ denotes its cumulative distribution function (cdf), $\tilde{x}(s)$ denotes the Laplace Stieltjes Transform of $X(x)$, and $\tilde{X}(s)$ denotes the Laplace Transform of $X(x)$. If $X$ is discrete then $x(k)$ denotes its probability mass function (pmf), and $\tilde{x}(z)$ its probability generating function (pgf).

\section{ASYMPTOTIC $(N \rightarrow \chi)$ DELAY ANALYSIS FOR THE PRIORITY CONTENTION RESOLUTION SCHEME}

A fraction $\alpha$ of the inputs carry type 1 traffic with burst length parameter $p_{1}$ and the remaining inputs carry type 2 traffic with parameter $p_{2}$. HOL contention is always resolved in favor of type 1 inputs. Random selection is used within a type. Recall that if $p_{1}<p_{2}$ we have the SEBF scheme, and if $p_{1}>p_{2}$ we have LEBF scheme. We do the queue length analysis for infinite input buffers, and for the asymptotic case $(N \rightarrow \infty)$.

Before any delay analysis can begin, we need to obtain the saturation throughput of the switch, and establish a stability condition for the queue length processes. We have performed a detailed saturation throughput analysis of the input queuing switch with multiclass traffic, as modeled above (see [7]). We have obtained closed form expressions for the asymptotic $(N \rightarrow \infty)$ saturation throughput of high and low priority cells. Further, we have argued in [8] that saturation throughput yields a sufficient condition for the stability of the input queues; i.e.. if the arrival rate at each input queue is less than the rate 
at which cells are served from that input, if the queues were saturated, then the input queues will be stable. In the analysis presented in this paper, it is assumed that the stability condition is met, and in all the numerical results, the parameters are chosen so that the input queues are stable.

If we focus on all the HOL cells of both type 1 and type 2 input queues destined for a particular output, we can consider them as belonging to a logical queue. The queue length process of this logical queue is a 2-D stochastic process representing the number of type 1 and type 2 HOL cells contending for the particular output. From this logical queue, in each slot, either a type $1 \mathrm{HOL}$ cell is randomly chosen for service or a type $2 \mathrm{HOL}$ cell is chosen (if there are no type $1 \mathrm{HOL}$ cells in this queue). Thus, associated with each output there will be a logical queue of HOL cells; this is called an HOL contention process.

Burst delay at an input queue is defined as the time from the first cell of the burst arriving at the input buffer, until the last cell of the burst is transmitted across the switch fabric to the output link. Burst delay has two components; the first is the time from when the first cell of the burst arrives at the input buffer, until this cell reaches the head of the input queue. This is the burst waiting time. When the leading cell of the burst reaches the head of the queue, it joins the HOL contention process associated with the output to which this burst is destined, and begins to contend for transmission to its designated output link. Once this cell is transmitted, the subsequent cells of the burst move to the head of the input queue to contend, in turn, for the same output until the last cell of the burst is transmitted. Thus, the second component of the burst delay is the sojourn time of the entire burst in an HOL contention process. This is called the HOL contention delay.

\section{A. High Priority Burst Delay}

We focus on a type 1 input queue (there are $\alpha N$ such queues), henceforth called the tagged input queue. The presence of low priority traffic on type 2 inputs is transparent to the high priority traffic. Let $\gamma_{1}$ be the cell arrival rate at each type 1 input.

Consider a tagged burst in the tagged input queue. Let $S_{H}$ denote the HOL contention delay of this burst. We obtain the pgf of $S_{H}$. Focus on the tagged output to which the cells of this tagged burst are destined. We can view the HOL cells contending for this output as each representing an (HOL) burst at their respective inputs.

Let $k(\geq 0)$ index the slots. Consider the epoch of the boundary between two slots. Just prior to this epoch, one burst, among those queued for the tagged output, is selected at random and the first cell of the burst is transmitted. The same is done for the other outputs also. Now some bursts will have cells remaining in them and will continue to contend in the same contention processes, whereas for some bursts the last cell has just been transmitted. At the inputs corresponding to the latter category of bursts, new bursts may be waiting. These will result in arrivals to the HOL contention processes. Let $H_{k}$ denote the number of $\mathrm{HOL}$ cells in the tagged output contention process just after slot $k$ (the start of slot $k+1)$ but prior to new HOL burst arrivals. If the HOL burst corresponding to a cell just served has not completed then this burst is included in $H_{k}$. Clearly

$$
H_{k+1}=\left(H_{k}+A_{k+1}-Z_{k}\right)^{+}
$$

where $A_{k+1}$ is the number of new HOL burst arrivals (into the tagged output contention process) at the beginning of slot $k+1$, and

$$
Z_{k}= \begin{cases}0, & \text { w.p. } p_{1} \\ 1, & \text { w.p. } 1-p_{1}\end{cases}
$$

In the asymptotic case, the process $\left\{A_{k}\right\}$ of arrivals of the first cells of bursts from other high priority inputs to the tagged HOL contention process is a sequence of independent Poisson random variables with mean $\alpha \lambda_{1}$, where $\lambda_{1}$ is the burst arrival rate at the high priority input queues $\left(\lambda_{1}=\gamma_{1}\left(1-p_{1}\right)\right)$ [8]. The HOL contention process can thus be modeled as a $B D / D / I$ queue with feedback, where Batch Deterministic $(B D)$ stands for the (deterministic) arrival of a (Poisson) batch of bursts at the beginning of every time slot, $D$ stands for the one slot service time of a cell, and the feedback occurs with probability $p_{1}$. Let $H$ denote the stationary random variable for $\left\{H_{k}\right\}$. With standard techniques $[11, \mathrm{Sec} .5 .6]$, we obtain

$$
\tilde{h}(z)=\frac{(1-z)\left(1-p_{1}-\alpha \lambda_{1}\right)}{\left[1-p_{1}(1-z)\right] e^{-\alpha \lambda_{1}(1-z)}-z} .
$$

1) PGF of High Priority HOL Contention Delay: Since the process of arrivals of new HOL bursts to the tagged contention process is a sequence of independent and identically distributed (i.i.d.) (Poisson) random variables, the HOL contention time of the tagged high priority burst $\left(S_{H}\right)$ is the time to absorption in a Markov chain. The state of this Markov chain is the number of HOL bursts (including the tagged burst) contending for the output. Recall that there is only one cell from the tagged burst in the tagged HOL contention process at any time. In each state of the Markov chain there is a probability that the cell from the tagged burst is selected for service, and that this is the last cell of the burst. This is the probability of absorption. Thus the absorbing state of the Markov chain corresponds to the successful transmission of the last cell of the tagged burst. This Markov chain has an infinite state space. We truncate the state space by assuming that there will not be more than $m_{1}$ bursts in the HOL contention process at any time and the error due to this truncation of state space is reduced to a negligibly small value by making $m_{1}$ sufficiently large. Thus we have an $\left(m_{1}+1\right)$-state Markov chain with transition probability matrix

$$
\mathbf{P}=\left|\begin{array}{cc}
\mathbf{R} & \mathbf{R}_{0} \\
\mathbf{0} & 1
\end{array}\right|
$$

where $\mathbf{R}$ is an $m_{1} \times m_{1}$ substochastic matrix, such that $\mathbf{I}-\mathbf{R}$ is nonsingular ( $I$ is the identity matrix). The initial probability vector is $\left(\mathbf{y}, y_{m+1}\right)$ (given below). It now follows that $S_{H}$ has discrete phase type distribution ( $\mathrm{PH}$-distribution) with representation $(\mathbf{y}, \mathbf{R})[16$, Sec. 2.2]. Letting $c(n)$ denote the pmf of the batch size of the new bursts arriving at the HOL 
process at the beginning of each time slot, the elements of $\mathbf{R}$, i.e., $r_{i j}, 1 \leq i . j \leq m_{1}$, are found to be

$$
\begin{aligned}
& r_{i j}= \\
& \left\{\begin{array}{ll}
\frac{i-1}{i}\left(1-p_{1}\right) r(0) . & 1<i \leq m_{1}, \text { if } j=i-1 \\
p_{1} c(j-i)+\frac{i-1}{i}\left(1-p_{1}\right) & \\
r(j-i+1) . & \text { if } j \geq i .1 \leq i . j \leq m_{1} \\
0 . & \text { otherwise }
\end{array},\right.
\end{aligned}
$$

where

$$
c(n)=\frac{e^{-\alpha \lambda_{1}}\left(\alpha \lambda_{1}\right)^{n}}{n !}
$$

Further

$$
\mathbf{y}=\left[y(n) .1 \leq n \leq m_{1}\right]
$$

where

1) $n$ HOL bursts, including itself

$\mathbf{y}$ is obtained from the stationary distribution of $\left\{H_{k}\right\}$, and the distribution of the size of the batch of bursts in which the tagged burst arrives at the output contention process.

Let, for $n \geq 1, \nu(n)$ be batch biased probability that tagged burst arrives in a batch of bursts of size $n$

Then [17, Sec. 2.5]

$$
\begin{aligned}
\nu(n) & =\frac{n c(n)}{\sum_{j=1}^{\infty} j c(j)} \\
& =\frac{e^{-\alpha \lambda_{1}}\left(\alpha \lambda_{1}\right)^{n-1}}{(n-1) !} .
\end{aligned}
$$

Since the bursts arrive in i.i.d. batches (that are Poisson distributed), the process indicating whether or not a batch is empty is a Bernoulli process. Hence we can make use of the discrete time version of Poisson Arrivals See Time Averages (PASTA), i.e., Geometric Arrivals See Time Averages (GASTA) [4], to assert that the stationary distribution of the number of HOL bursts found by a nonempty batch of bursts is equal to the stationary distribution of $\left\{H_{k}\right\}$. Hence, $y(n)$ can be computed as the convolution sum of $h(n)$ and $\nu(n)$

$$
y(n)=\sum_{i=0}^{n-1} \nu(n-i) h(i) . \quad n \geq 1 .
$$

Note that $h(n)$ is the pmf of $H$, and it is obtained from $\tilde{h}(z)$ (1) as

$$
h(n)=\lim _{z \rightarrow 0} \frac{1}{n !} \frac{d^{(n)}}{d z} \tilde{h}(z) .
$$

We need only a finite number of terms of this distribution because of the truncation of the state space mentioned above. Finally, since $S_{H}$ has discrete phase type distribution $(\mathbf{y}, \mathbf{R})$, we get the pgf of $S_{H}$ as [16]

$$
\grave{s}_{H}(z)=z \mathbf{y}(\mathbf{I}-z \mathbf{R})^{-1} \mathbf{R}_{0} .
$$

2) Mean High Priority Burst Delay: Observe that the HOL contention delay $S_{H}$ of a burst can be viewed as its effective service time at the input queue. In the asymptotic case (i.e., $N \rightarrow \infty)$, the bursts destined for the same output link are separated far apart (in time) on an input link so that the HOL contention delays of these bursts can be considered to be independent of each other. Also, as $N \rightarrow x$, the HOL contention processes for different outputs tend to be mutually independent [8]. Thus the HOL contention delays of successive bursts in an input buffer can be considered to be a sequence of i.i.d. random variables, which allows us to model the tagged input buffer as a ./GI/l queue. $\mathrm{Li}$ [12] has argued similarly that. in the limit $(N \rightarrow \infty)$, each input queue can be considered as an independent queue with an i.i.d. service sequence.

The actual cell arrival process on the tagged input, described in Section I, results in a cell arrival rate (in cells per slot) $\gamma_{1}=\left(1-q_{1}\right) /\left(1-p_{1} q_{1}\right)$, or alternatively, a burst arrival rate $\lambda_{1}=\gamma_{1}\left(1-p_{1}\right)$. For analytical simplicity we approximate the burst arrival process (i.e., the point process of the arrival epochs of the leading cells of the bursts) at the tagged input queue by a Bernoulli process with rate $\lambda_{1}$, and assumes that a batch of cells constituting a burst arrive in a single slot. Note that the actual burst interarrival time is the sum of a geometric burst duration (at least one slot) and a geometric idle period (possibly zero slot). We expect that the assumption of geometric interarrival time will lead to an over estimation of the burst delay. Note that if the interarrival times are modeled exactly the batch arrival assumption in itself does not have any effect on the burst delay since the time it takes to transmit a cell across the switch is the same as the time it takes for a cell to arrive on the input link; so the next cell in the burst is already in the input buffer when the previous cell is fully transmitted.

Thus the tagged input is modeled as a Geom/GL/1 queue. Observe that the service time in this model, i.e., the HOL contention delay of the burst, is insensitive to the assumptions made above since it takes at least one slot for each HOL cell to get transmitted onto the output link.

Using the known results for the Geom/GI/1 queue [15] we obtain the mean waiting time for the tagged burst at the input queue. Let $W_{H}$ denote the time from the arrival of the tagged burst, until it reaches the head of the queue. Then

$$
E W_{H}=\frac{\lambda_{1} E\left[S_{H}\left(S_{H}-1\right)\right]}{2\left(1-\lambda_{1} E S_{H}\right)}
$$

where $E S_{H}$ and $E\left[S_{H}\left(S_{H}-1\right)\right]$ are obtained from (3) as

$$
\begin{aligned}
E S_{H} & =\mathbf{y}(\mathbf{I}-\mathbf{R})^{-1} \mathbf{e} \\
E\left[S_{H}\left(S_{H}-1\right)\right] & =2 \mathbf{y} \mathbf{R}(\mathbf{I}-\mathbf{R})^{-2} \mathbf{e}
\end{aligned}
$$

where $\mathbf{e}$ is the unit vector.

The mean burst delay at a high priority input queue is given by $E W_{H}+E S_{H}$. The mean burst delay that we compute with the Geom/GI/1 model should be an upper bound to the actual value, as the squared coefficient of variation of the actual burst interarrival time is less than that of the assumed geometric interarrival time. This observation is borne out by the simulation results presented in Section III. 


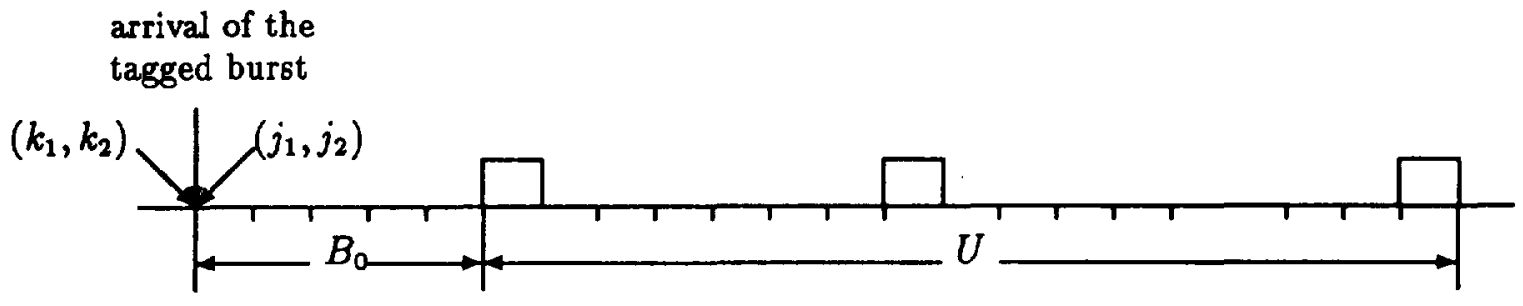

Fig. 3. Schematic representation of the low priority burst sojourn time in an HOL contention queue.

\section{B. Low Priority Burst Delay}

Now we focus on a type 2 input queue, to which arrives low priority traffic with a different burst length parameter, $p_{2}$. Let the cell arrival rate be $\gamma_{2}$ and the corresponding burst arrival rate at the input be $\lambda_{2}$. Recall that there are $(1-\alpha) N$ type 2 input queues.

First we compute the pgf of the HOL contention delay $S_{L}$ of a low priority burst at the tagged input queue. The tagged low priority burst moves to the head of the queue and joins an HOL contention process. Consider the HOL contention process that this burst joins. In the asymptotic case $(N \rightarrow \infty)$, independent Poisson batches of high priority and low priority HOL bursts with mean $\alpha \lambda_{1}$ and $(1-\alpha) \lambda_{2}$, respectively, arrive at the tagged HOL contention process at the beginning of each time slot. A low priority cell in the HOL contention process is selected for transmission, only if there are no high priority cells in that process. It appears at first that in order to analyze $S_{L}$ we will need to analyze the 2-D process of low and high priority bursts in the contention process. The mean of $S_{L}$ (i.e., $E S_{L}$ ) can be obtained, however, by a direct method, as we show in the Appendix.

1) PGF of Low Priority HOL Contention Delay: Consider the tagged low priority burst. When it arrives in its HOL contention process, it observes an ongoing high priority busy period (see Fig. 3 ). The residual busy period $B_{0}$ is determined by the number of high priority bursts already there at the time of arrival of the tagged low priority burst, and the batch size of the HOL high priority bursts arriving into the same contention process along with the tagged low priority burst. The number of low priority and high priority bursts in the HOL contention process are dependent. Therefore we need the joint probability distribution of the number of high priority and low priority bursts in the HOL contention process.

Let $\mathbf{X}_{k}=\left[X_{k}^{(1)}, X_{k}^{(2)}\right]$, where $X_{k}^{(1)}$ [respectively, $\left.X_{k}^{(2)}\right]$ is the number of high priority (respectively low priority) bursts in the HOL contention process just after slot $k$ but prior to new HOL burst arrivals. If the HOL burst corresponding to the cell just served has not completed then this burst is included in $\mathbf{X}_{k}$. Clearly $\left\{\mathbf{X}_{k}, k \geq 0\right\}$ is a 2-D homogeneous Markov chain. Let the transition probabilities of this Markov chain be denoted by $p_{i_{1} i_{2}, j_{1} j_{2}}$, i.e.,

$$
\begin{aligned}
& p_{i_{1} i_{2}, j_{1} j_{2}}= \\
& \quad \operatorname{Pr}\left[X_{n+1}^{(1)}=j_{1}, X_{n+1}^{(2)}=j_{2} / X_{n}^{(1)}=i_{1}, X_{n}^{(2)}=i_{2}\right] .
\end{aligned}
$$

Let $\mathbf{X}=\left[X^{(1)}, X^{(2)}\right]$ be the stationary random vector for $\left\{\mathbf{X}_{k}\right\}$ and $x\left(k_{1}, k_{2}\right)$ be the joint probability distribution of $\mathbf{X}$. With $C_{1}$ and $C_{2}$ as defined earlier, $\bar{p}_{i}$ denoting $1-p_{i}$, $i=1,2$, we have the following set of equations for the transition probabilities

$$
\begin{aligned}
& p_{00,00} \\
& =c_{1}(0) c_{2}(\mathbf{0})+c_{1}(1) c_{2}(0) \bar{p}_{1}+c_{1}(0) c_{2}(\mathbf{1}) \bar{p}_{2} \\
& p_{0 n_{2}, 0\left(n_{2}-1\right)} \\
& =c_{1}(0) c_{2}(0) \bar{p}_{2} ; \quad n_{2}>0 \\
& p_{0 n_{2}, 0\left(n_{2}+m_{2}\right)} \\
& =c_{1}(1) c_{2}\left(m_{2}\right) \bar{p}_{1}+c_{1}(0) c_{2}\left(m_{2}\right) p_{2} \\
& \quad+c_{1}(0) c_{2}\left(m_{2}+1\right) \bar{p}_{2} ; \\
& \quad n_{2}>0, m_{2} \geq 0 \quad \text { or } \quad n_{2} \geq 0, m_{2}>0 \\
& p_{n_{1} n_{2},\left(n_{1}-1\right)\left(n_{2}+m_{2}\right)} \\
& =c_{1}(0) c_{2}\left(m_{2}\right) \bar{p}_{1} ; \quad n_{1}>0, n_{2}, m_{2} \geq 0 \\
& p_{n_{1} n_{2},\left(n_{1}+m_{1}\right)\left(n_{2}+m_{2}\right)} \\
& =c_{1}\left(m_{1}\right) c_{2}\left(m_{2}\right) p_{1}+c_{1}\left(m_{1}+1\right) c_{2}\left(m_{2}\right) \bar{p}_{1} ; \\
& \quad n_{1}, n_{2}, m_{2} \geq 0, m_{1}>0 \\
& \quad \text { or } \quad n_{2}, m_{1}, m_{2} \geq 0, n_{1}>0 .
\end{aligned}
$$

The equilibrium joint distribution is given by

$$
\begin{gathered}
x\left(k_{1}, k_{2}\right)=\sum_{\substack{l_{1}=0 \\
k_{1}, k_{2} \geq 0 .}}^{\infty} \sum_{l_{2}=0}^{\infty} x\left(l_{1}, l_{2}\right) p_{l_{1} l_{2}, k_{1} k_{2}} \\
\text {. }
\end{gathered}
$$

Now defining the 2-D generating function

$$
\tilde{x}\left(z_{1}, z_{2}\right)=\sum_{k_{1}=0}^{\infty} \sum_{k_{2}=0}^{\infty} z_{1}^{k_{1}} z_{2}^{k_{2}} x\left(k_{1}, k_{2}\right)
$$

and applying it to (4), we obtain after some manipulations

$$
\begin{aligned}
\tilde{x}\left(z_{1}, z_{2}\right)=\{ & \left\{\left(\tilde{x}_{0}\left(z_{2}\right) c_{1}(0) \tilde{c}_{2}\left(z_{2}\right)\right.\right. \\
& \cdot\left[z_{1}\left(\bar{p}_{2}+p_{2} z_{2}\right)-z_{2}\left(\bar{p}_{1}+p_{1} z_{1}\right)\right] \\
& \left.+x(0,0) z_{1} c_{1}(0) c_{2}(0) \bar{p}_{2}\left(z_{2}-1\right)\right\} / \\
& \left\{z_{2}\left[z_{1}-\tilde{c}_{1}\left(z_{1}\right) \tilde{c}_{2}\left(z_{2}\right)\left(\bar{p}_{1}+p_{1} z_{1}\right)\right]\right\}
\end{aligned}
$$

where

$$
\tilde{x}_{0}\left(z_{2}\right)=\sum_{k_{2}=0}^{\infty} x\left(0, k_{2}\right) z_{2}^{k_{2}} .
$$

To solve for the unknown function $\tilde{x}_{0}\left(z_{2}\right)$, we equate $\lim _{z_{1} \rightarrow 1} \tilde{x}\left(z_{1}, z_{2}\right)$ to the generating function of the marginal distribution of the number of low priority bursts. Noting that the marginal process $\left\{X_{k}^{(2)}\right\}$ is just $\left\{X_{k}\right\}$ defined in the Appendix, we have

$$
\lim _{z_{1} \rightarrow 1} \tilde{x}\left(z_{1}, z_{2}\right)=\tilde{x}\left(z_{2}\right)
$$


where,$\left.\hat{r}_{2}\right)$ is given by (A.9) in the Appendix. This yields

$$
\begin{aligned}
& \dot{r}_{0}\left(z_{2}\right)= \\
& \quad \frac{E C_{2}\left(1-p_{2}-E \Gamma\right) z_{2}\left[1-\dot{\gamma}\left(z_{2}\right)\right]}{E \Gamma\left[j\left(z_{2}\right)\left(\bar{p}_{2}+p_{2} z_{2}\right)-z_{2}\right]}+x(0,0) c_{1}(0) c_{2}(0) \bar{p}_{2} \\
& \frac{c_{1}(0)\left(1-p_{2}\right) \tilde{c}_{2}\left(z_{2}\right)}{}
\end{aligned}
$$

The unknown quantity $x(0.0)$ is obtained by applying the normalizing condition, i.e..

$$
\lim _{z=1} \lim _{z: \rightarrow 1} \tilde{x}\left(z_{1}, z_{2}\right)=1
$$

Thus, we get

$$
c_{1}(0) c_{2}(0) r(0.0)=1-\frac{E C_{1}}{1-p_{1}}-\frac{E C_{2}}{1-p_{2}} .
$$

Observe that the left hand side of the above formula is the fraction of idle slots at the output corresponding to the tagged HOL process. Since $E C_{1}$ and $E C_{2}$ are high and low priority burst arrival rates into this HOL process, and $1 /\left(1-p_{1}\right)$ and $1 /\left(1-p_{2}\right)$ are the respective mean burst lengths, this formula is intuitively correct.

An application of GASTA again yields that the joint probability distribution $r\left(k_{1}, k_{2}\right)$ is also the joint probability distribution of the number of high priority and low priority HOL bursts as found by a nonempty batch of low priority HOL bursts on its arrival.

Let $r\left(j_{1}, j_{2}\right)$ be the probability that the tagged low priority HOL burst starts contention with $j_{1}$ high priority HOL bursts and $j_{2}$ low priority HOL bursts. It is clear that (see Fig. 3)

$$
r\left(j_{1} \cdot j_{2}\right)=\sum_{k_{2}=0}^{j_{2}-1} \sum_{k_{1}=0}^{j_{1}} r\left(k_{1}, k_{2}\right) c_{1}\left(j_{1}-k_{1}\right) \hat{c}_{2}\left(j_{2}-k_{2}\right)
$$

where $\hat{r}_{2}(n)=\operatorname{Pr}$ (batch size of low priority burst bringing the tagged one into the HOL contention process is $n$ ); this will be a batch size biased probability, i.e., $\hat{c}_{2}(n)=\left[n c_{2}(n) / E C_{2}\right]$.

In Fig. 3, $B_{0}$ is the residual high priority busy period as observed by the tagged low priority burst on its arrival into the HOL contention process, and $U$ is the remaining sojourn time of the tagged burst in the HOL process. Thus

$$
S_{L}=B_{0}+U \text {. }
$$

We now analyze the random variable $U$. Let $\left\{M_{k}, k \geq 0\right\}$ denote the number of low priority bursts (in the tagged HOL process) at the beginning of the $(k+1)$ st low priority cell service during the period $U$. Thus $M_{0}$ is the number of low priority bursts at the beginning of $U$ (see Fig. 3). Let $\left\{T_{k}, k \geq 0\right\}$ denote the number of slots between successive low priority cell services during the period $U$. Observe that $\left\{\left(M_{k}, T_{k}\right) . k \geq 0\right\}$ is a Markov Renewal process, and $U$ is the time to "absorption" in this process, where absorption corresponds to the successful transmission of the last cell of the tagged low priority burst. Each time a cell is served, there is a probability that the cell from the tagged low priority burst is selected for service, and that this is the last cell of the tagged burst. This is the probability of absorption. We truncate the state space by assuming that there will not be more than $m_{2}$ low priority bursts in the tagged HOL contention process at any time.

Consider the discrete time Markov Renewal Process $\left\{\left(M_{k}, T_{k}\right), k \geq 0\right\}$ with transition probability matrix for the Markov chain $\left\{M_{k}, k \geq 0\right\}$ of the form

$$
\mathbf{P}=\left|\begin{array}{cc}
\mathbf{Q} & \mathbf{Q}_{0} \\
\mathbf{0} & \mathbf{1}
\end{array}\right|
$$

where $\mathbf{Q}$ is an $m_{2} \times m_{2}$ matrix and $\mathbf{Q}_{0}$ is an $m_{2} \times 1$ vector. Let $\alpha$ denote the absorbing state.

Using the random variable $\Gamma$ from the Appendix, clearly, the elements of $\mathbf{Q}$, i.e., $q_{i j} .1 \leq i . j \leq m_{2}$, are given by

$$
\begin{aligned}
& q_{i j}= \\
& \left\{\begin{array}{cl}
\frac{(i-1)}{i}\left(1-p_{2}\right) \gamma(0) . & \text { if } j=i-1 \\
p_{2} \gamma(j-i)+\frac{i-1}{i} & \\
\cdot\left(1-p_{2}\right) \gamma(j-i+1), & \text { if } j \geq i \\
0 . & \text { otherwise }
\end{array}\right.
\end{aligned}
$$

and the elements of the vector $\mathbf{Q}_{0}$ by

$$
q_{i \alpha}=\frac{1}{i}\left(1-p_{2}\right): \quad 1 \leq i \leq m_{2} \text {. }
$$

Observe that

$$
\begin{aligned}
& \operatorname{Pr}\left(T_{k}=n \mid M_{k+1}=j \cdot M_{k}=i\right)= \\
& \frac{\operatorname{Pr}\left(T_{k}=n \mid M_{k}=i\right) \operatorname{Pr}\left(M_{k+1}=j \mid T_{k}=n, M_{k}=i\right)}{\operatorname{Pr}\left(M_{k+1}=j \mid M_{k}=i\right)}
\end{aligned}
$$

Let $L_{i j}$ be a random variable with this pmf, i.e.,

$$
l_{i j}(n):=\operatorname{Pr}\left(T_{k}=n \mid M_{k+1}=j, M_{k}=i\right)
$$

and denote by $i_{i j}(z)$ the corresponding pgf. Also, denote by $U^{(i)}$ the time to absorption with the initial state $i$, i.e., $M_{0}=i$. Then

$$
\tilde{u}^{(i)}(z)=q_{i \gamma} \tilde{l}_{i \gamma}(z)+\sum_{j \neq \alpha} q_{i j} \tilde{l}_{i j}(z) \tilde{u}^{(j)}(z)
$$

Since the sojourn time in the last state before absorption is 1 slot with probability 1 (see Fig. 3 ), $\tilde{l}_{i \alpha}(z)=z$ and we have

$$
\tilde{u}^{(i)}(z)=z q_{i \kappa}+\sum_{j \neq \alpha} q_{i j} \tilde{l}_{i j}(z) \tilde{u}^{(j)}(z) .
$$

Setting

$$
\check{\phi}_{i, x}(z)=z q_{i \alpha}
$$

and

$$
\tilde{\phi}_{i j}(z)=q_{i j} \tilde{l}_{i j}(z)
$$

and using the vector notation, (7) can be written as

$$
\begin{aligned}
\tilde{\mathbf{u}}(z) & =\tilde{\boldsymbol{\Phi}}_{0}(z)+\tilde{\boldsymbol{\Phi}}(z) \tilde{\mathbf{u}}(z) \\
& =[\mathbf{I}-\tilde{\boldsymbol{\Phi}}(z)]^{-1} \tilde{\boldsymbol{\Phi}}_{0}(z)
\end{aligned}
$$

where the $m_{2} \times 1$ vector $\dot{\boldsymbol{\Phi}}_{0}(z)=\left[\tilde{\boldsymbol{\Phi}}_{\text {i⿱ }}(z)\right]$ and the $m_{2} \times m_{2}$ matrix $\check{\boldsymbol{\Phi}}(z)=\left[\tilde{\boldsymbol{\Phi}}_{i j}(z)\right]$. $(\mathbf{I}-\mathbf{Q})$ is nonsingular, and hence the inverse in the above formula exists. 
Now we are ready to obtain the generating function of the HOL contention delay $S_{L}$ of the tagged low priority burst. With reference to Fig. 3, we can write

$$
\tilde{s}_{L}(z)=\sum_{n=1}^{m_{2}} \sum_{l=0}^{\infty} p(l, n) z^{l} \tilde{u}^{(n)}(z)
$$

where $\tilde{u}^{(n)}(z)$ is given by the $n$th component of the vector $\tilde{\mathbf{u}}(z)(8)$ and $p(l, n)=\operatorname{Pr}\left(B_{0}=l, M_{0}=n\right)$. Recall that $B_{0}$ is the residual high priority busy period seen by the arriving tagged low priority burst and $M_{0}$ is the initial state of the $\left(m_{2}+1\right)$ state Markov chain $\left\{M_{k}, k \geq 0\right\}$ defined above.

Define

1) $B_{0}^{(n)}:=$ busy period initiated by $n$ high priority bursts,

2) $\Gamma^{(l)}:=$ total number of low priority bursts arriving at the tagged HOL process during $l$ time slots.

$$
\text { Clearly }
$$

$$
p(l, n)=\sum_{j_{2}=1}^{n} \sum_{j_{1}=0}^{m_{1}} r\left(j_{1}, j_{2}\right) b_{0}^{\left(j_{1}\right)}(l) \gamma^{(l)}\left(n-j_{2}\right)
$$

where $r\left(j_{1}, j_{2}\right)$ is given by (6). As mentioned earlier we assume that not more than $m_{1}$ high priority bursts will be present in an HOL contention process at any time, which amounts to truncating the second summation in the above equation to $m_{1}$. It is obvious that the pgf of $\Gamma^{(l)}$ is

$$
\tilde{\gamma}^{(l)}(z)=e^{-l(1-\alpha) \lambda_{2}(1-z)} \text {. }
$$

To obtain the pgf of $B_{0}^{(n)}$, note first that

$$
\tilde{b}_{0}^{\left(j_{1}\right)}(z)=\left[\tilde{b}_{0}^{(1)}(z)\right]^{j_{1}}
$$

and the pgf of the service time of high priority cells constituting a high priority burst is $z\left(1-p_{1}\right) /\left(1-p_{1} z\right)=: \tilde{\psi}(z)$. Moreover independent Poisson batches of high priority bursts are arriving in every time slot. It is easily seen that

$$
\tilde{b}_{0}^{(1)}(z)=\bar{\psi}\left\{z \tilde{c}_{1}\left(\tilde{b}_{0}^{(1)}(z)\right) .\right.
$$

Finally, substituting $p(l, n)(10)$ and $\tilde{u}^{(n)}(z)$ (7) in (9), we obtain the pgf of the HOL contention delay of the tagged low priority burst, $\tilde{s}_{L}(z)$. To examine the effect of the truncation, later we will compare the mean value $E S_{L}$ obtained from $\tilde{s}_{L}(z)$ with that found in the Appendix by (A.10).

2) Mean Low Priority Burst Delay: Following the discussion of Section II-A, the tagged low priority input queue is modeled as a Geom/GI/1 queue in order to compute the mean burst waiting time. Again, using similar notations as in Section II-A, mean delay of a low priority burst is given by

$$
E W_{L}+E S_{L}=\frac{\lambda_{2} E\left[S_{L}\left(S_{L}-1\right)\right]}{2\left(1-\lambda_{2} E S_{L}\right)}+E S_{L}
$$

where $E S_{L}$ and $E\left[S_{L}\left(S_{L}-1\right)\right]$ are obtained from (9) as

$$
\begin{aligned}
E S_{L}= & \sum_{n=1}^{m_{2}} \sum_{l=0}^{\infty} p(l, n)\left[l \tilde{u}^{(n)}(1)+\tilde{u}^{(n)^{\prime}}(1)\right] \\
E\left[S_{L}\left(S_{L}-1\right)\right]= & \sum_{n=1}^{m_{2}} \sum_{l=0}^{\infty} p(l, n)\left[l(l-1) \tilde{u}^{(n)}(1)\right. \\
& \left.+2 l \tilde{u}^{(n)^{\prime}}(1)+\tilde{u}^{(n)^{\prime \prime}}(1)\right] .
\end{aligned}
$$

TABLE I

Mean hol Contention Delay for low Priority Burst Computed From Two METHODS; $E S_{L}^{(1)}$ IS FROM (A.10) AND $E S_{L}^{(2)}$ IS From THE Generating Function $(11) ; p_{1}=0.3, p_{2}=0.7, \gamma_{2}=0.2$

\begin{tabular}{c|c|c|c|c|c}
\hline \multirow{2}{*}{$\gamma_{1}$} & \multicolumn{2}{|c|}{$S E B F$} & & \multicolumn{2}{c}{$L E B F$} \\
\cline { 2 - 6 } & $E S_{L}^{(1)}$ & $E S_{L}^{(2)}$ & & $E S_{L}^{(1)}$ & $E S_{L}^{(2)}$ \\
\hline \hline .1 & 3.891 & 3.889 & & 1.963 & 1.91 \\
.2 & 4.171 & 4.164 & & 2.054 & 1.999 \\
.3 & 4.496 & 4.475 & & 2.158 & 2.097 \\
.4 & 4.879 & 4.823 & & 2.276 & 2.2 \\
.5 & 5.335 & 5.210 & & 2.413 & .2 .305 \\
\hline
\end{tabular}

Note that $\tilde{u}^{(n)^{\prime}}(1)$ and $\tilde{u}^{(n)}(1)$ are the elements of the vectors $\tilde{\mathbf{u}}^{\prime}(1)$ and $\tilde{\mathbf{u}}^{\prime \prime}(1)$, respectively. We obtain $\tilde{\mathbf{u}}^{\prime}(z)$ and $\tilde{\mathbf{u}}^{\prime \prime}(z)$ by differentiating $\tilde{\mathbf{u}}(z)$ (8). Thus

$$
\begin{aligned}
\tilde{\mathbf{u}}^{\prime}(z)= & {[\mathbf{I}-\tilde{\boldsymbol{\Phi}}(z)]^{-1} \tilde{\boldsymbol{\Phi}}^{\prime}(z)[\mathbf{I}-\tilde{\boldsymbol{\Phi}}(z)]^{-1} \tilde{\boldsymbol{\Phi}}_{0}(z) } \\
& +[\mathbf{I}-\tilde{\mathbf{\Phi}}(z)]^{-1} \tilde{\boldsymbol{\Phi}}_{0}^{\prime}(z) \\
\tilde{\mathbf{u}}^{\prime \prime}(z)= & 2\left\{[\mathbf{I}-\tilde{\mathbf{\Phi}}(z)]^{-1} \tilde{\boldsymbol{\Phi}}^{\prime}(z)\right\}^{2}[\mathbf{I}-\tilde{\mathbf{\Phi}}(z)]^{-1} \tilde{\boldsymbol{\Phi}}_{0}(z) \\
& +[\mathbf{I}-\tilde{\mathbf{\Phi}}(z)]^{-1} \tilde{\boldsymbol{\Phi}}^{\prime \prime}(z)[\mathbf{I}-\tilde{\mathbf{\Phi}}(z)]^{-1} \tilde{\boldsymbol{\Phi}}_{0}(z) \\
& +2[\mathbf{I}-\tilde{\mathbf{\Phi}}(z)]^{-1} \tilde{\boldsymbol{\Phi}}^{\prime}(z)[\mathbf{I}-\tilde{\mathbf{\Phi}}(z)]^{-1} \tilde{\boldsymbol{\Phi}}_{0}^{\prime}(z) \\
& +[\mathbf{I}-\tilde{\mathbf{\Phi}}(z)]^{-1} \tilde{\boldsymbol{\Phi}}_{0}^{\prime \prime}(z) .
\end{aligned}
$$

\section{NUMERICAL RESUltS: ANALYSIS AND SimUlation}

In getting the numerical results from the analysis, we have made some simplifications. As mentioned earlier we assume that not more than $m_{1}$ high priority bursts and $m_{2}$ low priority bursts will be present in an HOL contention process at any time. Further, we assume that the initial busy period as observed by the arriving tagged low priority burst will not be more than $m_{3}$ slots. This amounts to truncating the second summation in (9) to $m_{3}$. The numerical error due to these truncations is reduced to a negligibly small value by choosing sufficiently large values for the parameters $m_{1}, m_{2}$, and $m_{3}$.

We have used Mathematica [18] for numerical computation. We obtain the 2-D distributions $x\left(k_{1}, k_{2}\right)$, for $1 \leq k_{1} \leq m_{1}$ and $1 \leq k_{2} \leq m_{2}$, from the 2-D generating function $\tilde{x}\left(z_{1}, z_{2}\right)$ using the following fact:

$$
x\left(k_{1}, k_{2}\right)=\lim _{z_{1} \rightarrow 0} \lim _{z_{2} \rightarrow 0} \frac{1}{k_{1} ! k_{2} !} \frac{d^{\left(k_{1}\right)}}{d z_{1}} \frac{d^{\left(k_{2}\right)}}{d z_{2}} \tilde{x}\left(z_{1}, z_{2}\right) .
$$

To quantify the numerical error due to the above mentioned truncations, we compared $E S_{L}$ computed from (11) with that obtained from the closed form expression in (A.10). Tables I and II illustrate this comparison. Recall that in the SEBF policy the low priority traffic is type 2 with mean burst length $\left(1-p_{2}\right)^{-1}$, and in the LEBF policy the low priority traffic is type 1 with mean burst length $\left(1-p_{1}\right)^{-1}$. In the typical numerical illustrations of Tables I and II we have used $p_{1}=0.3, p_{2}=0.7, m_{1}=m_{2}=6$, and $m_{3}=8$. Table I shows the comparison with type 2 cell arrival rate $\gamma_{2}$ fixed at 0.2 , and Table II shows the results with type 1 cell arrival rate $\gamma_{1}$ fixed at 0.45 . The agreement is quite good and we accept these values of $m_{1}, m_{2}$, and $m_{3}$ as adequate.

Next we compare the analytical results with those from simulations. We recall that the analytical results are for switch 
TABLE II

mean hol Contention Delay for low Priority Blirst Computed FROM TWo METHODS; $E S(1) L$ IS FROM (A.10) AND $E S_{L}^{2}$ IS FROM THE Generating; Function $(11) ; p_{1}=0.3, p_{2}=0.7, i_{1}=0.45$

\begin{tabular}{c|c|c|c|c|c}
\hline \multirow{2}{*}{$\gamma_{2}$} & \multicolumn{2}{|c|}{$S E B F$} & \multicolumn{2}{c}{$L E B F$} \\
\cline { 2 - 6 } & $E S_{L}^{(1)}$ & $E S_{L}^{(2)}$ & & $E S_{L}^{(1)}$ & $E S_{L}^{(2)}$ \\
\hline \hline .05 & 4.637 & 4.627 & & 1.835 & 1.824 \\
.15 & 4.933 & 4.922 & & 2.155 & 2.101 \\
.25 & 5.274 & 5.261 & & 2.551 & 2.412 \\
.35 & 5.672 & 5.646 & & 3.049 & 2.731 \\
\hline
\end{tabular}

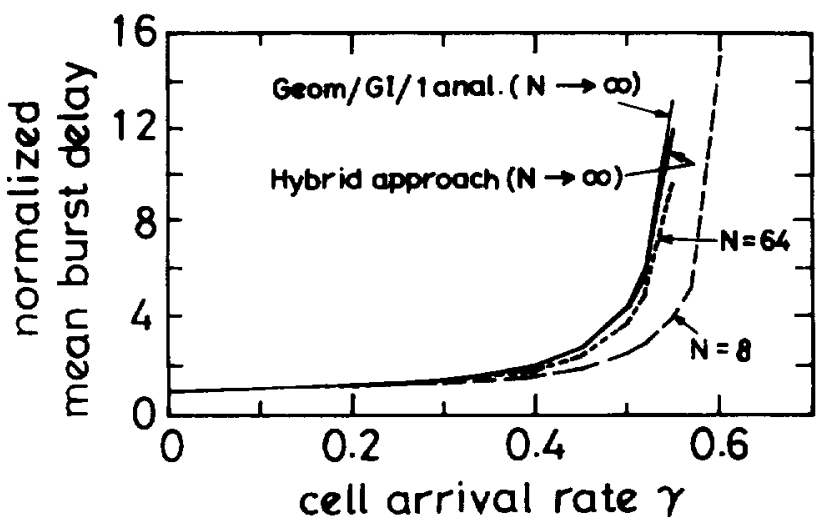

Fig. 4. Comparison of mean burst delay (normalized w.r.t. the mean burst length) for single elass traffic; $p=0.1$

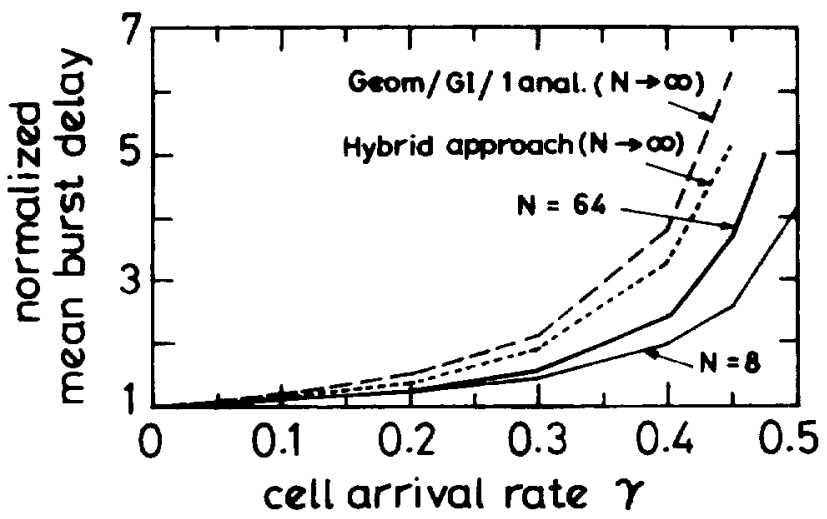

Fig. 5. Comparison of mean burst delay (normalized w.r.t. the mean burst length) for single class traffic: $p=0.6$

size $N \rightarrow \infty$. We have used SIMSCRIPT II.5 for simulation. Figs. 4-6 illustrate the comparison of normalized mean burst delay (normalized with respect to the mean burst length) obtained from the asymptotic analysis and from the simulation with different values for the switch size parameter $N$. These results are for single class traffic with burst length parameter of $0.1,0.6$, and 0.9 , respectively, i.e., mean burst lengths of $\frac{1}{0.9}, 2.5$, and 10 , respectively.

The curves labeled hybrid approach are obtained in the following way. The pgf of the effective service time of a burst in an input buffer (HOL contention time for the burst) is obtained by the asymptotic analysis of Section II-A. The distribution of the effective burst service time is then obtained by numerical inversion of the pgf [9]. We have simulated a single server FIFO queue with the service time distribution obtained in this

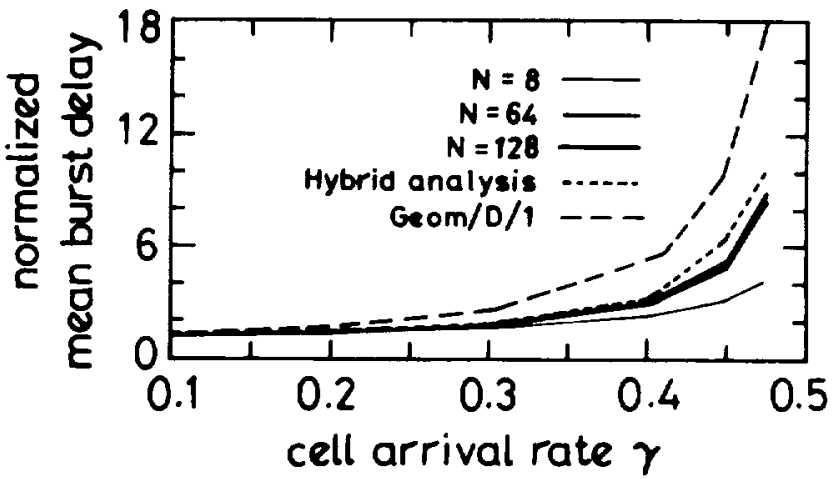

Fig. 6. Comparison of mean hurst delay (normalized w.r.t. the mean hurst length) for single class traffic: $y=0.9$.

manner, and with the actual burst arrival process as described in the Introduction. Recall that in the Geom/GI/I analysis we have used the geometric approximation for the burst arrival process. The hybrid approach reduces simulation time, and also captures the cell arrival process more accurately than the geometric approximation.

We observe from these figures that mean burst delay increases as $N$ increases, and the asymptotic analysis gives an upper bound. For Bernoulli traffic (i.e., $p=0$ in our model), it was observed in [10] that the mean cell delay for $N \geq 16$ is closely approximated by the asymptotic analysis $(N \rightarrow x)$. Note that in our work we have studied burst delays, which we believe are more appropriate for packetized isochronous traffic. Our results in Figs. 4-6 show, however, that progressively larger values of switch size $N$ are needed to come close to the asymptotic analysis as the burst length parameter $p$ increases. For $p=0.1$, the asymptotic analysis is a good approximation for $N=64$. For larger values of $p$, even for $N=64$ or $N=128$ the asymptotic analysis yields only an upper bound. The Geom/GI/1 analysis is close to the more accurate hybrid results for $p=0.1$, but becomes a loose upper bound for $p=0.6$ and $p=0.9$. The fact that Geom/GJ/1 yields an upper bound (even for the asymptotic mean delay) is as expected in our discussion accompanying the analysis (end of Section IIA). We note from Fig. 6 that the hybrid approach is a tighter upper bound for $N=128$, suggesting that for $p=0.9$ one needs to go upto $N=128$ to get close to the asymptotic results.

There is one important reason why asymptotic analysis can only be expected to yield upper bounds: in the asymptotic case. the input queues saturate at a lower arrival rate than for finite $N$ (see [8]). In [7], we have stated the following formula for the asymptotic saturation throughput $[T(p)]$ for the single class case, with each input carrying traffic of burstiness parameter $p$

$$
T(p)=\frac{2-\sqrt{\{4-2(1-p)\}}}{(1-p)} .
$$

Calculation using this formula yields $T(0.1)=0.574$, $T(0.6)=0.527$, and $T(0.9)=0.506$. We observe from Figs. 4-6 that, as expected, the switches with a finite number of inputs saturate at cell arrival rates higher than the asymptotic saturation throughput. 


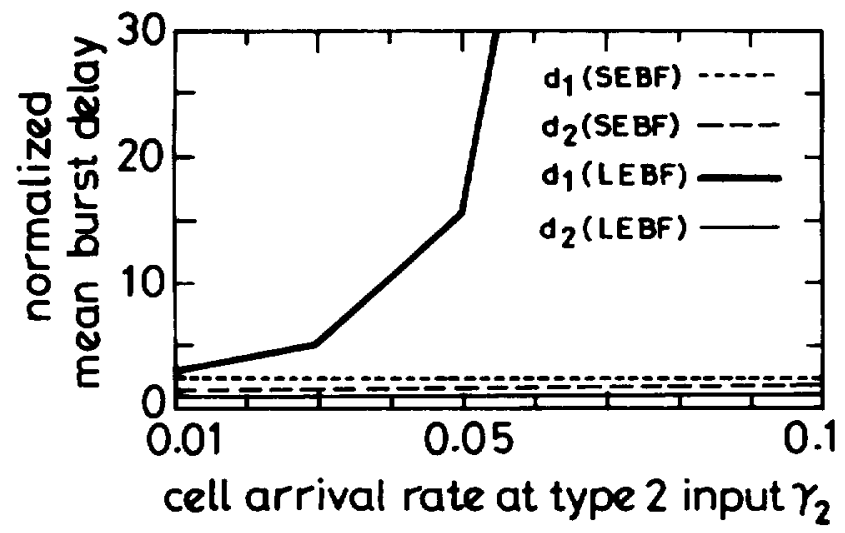

Fig. 7. Normalized mean burst delay for type 1 and type 2 traffic with the two priority selection contention resolution policies: analytical results $(N \rightarrow \infty)$ with $p_{1}=0.1, p_{2}=0.9 . \gamma_{1}=0.6 . a=0.5$.

We note that exact analysis for any reasonably large finite input queuing switch is intractable; asymptotic analysis becomes tractable because it is assumed that as $N \rightarrow \infty$ the arrival process into the output contention queues converges to Poisson (see Section II). Our results show that this more tractable analysis yields upper bounds on mean burst delay, and an accurate approximation for large switch sizes. We observe in the next section, however, that asymptotic analysis yields qualitative comparisons that continue to hold for finite switch sizes.

\section{Comparative Performance OF THE SCHEdUling StRategies}

In this section we compare the effect of SEBF, LEBF, and RS scheduling strategies on the mean burst delay, for a local access ATM switch with two classes of bursty traffic. Since the analysis of the switch with the RS scheme is intractable we have only simulation results (for finite $N$ ) for it, whereas for the SEBF and LEBF schemes we have both analytical (for $N \rightarrow \infty$ ) and simulation (for finite $N$ ) results. First we compare the two priority contention resolution policies, and seeing that the LEBF scheme is not desirable, we compare the SEBF with RS scheme. In the analytical as well as simulation results that we present here, we have fixed $\alpha$ (the fraction of switch inputs carrying type 1 traffic which is less bursty) to be 0.5 .

Figs. 7-12 illustrate the comparison of the normalized (with respect to mean burst length) mean burst delay with the two priority contention resolution policies, namely, SEBF and LEBF policies.

Figs. $7,8,11$, and 12 show the results with the cell arrival rate at type 1 inputs $\left(p_{1}=0.1\right.$ or $\left.p_{1}=0.9\right)$ kept at a constant value equal to 0.6 (which corresponds to type 1 throughput per output link of 0.3 ), and the cell arrival rate at type 2 inputs $\left(p_{2}=0.9\right.$ or $p_{2}=0.99$ ) varying. Figs. 7 and 11 give the asymptotic analysis results, whereas Figs. 8 and 12 give the simulation results for a finite switch with $N=16$.

Figs. 9 and 10 illustrate similar results with type 1 offered load varying and type 2 offered load fixed at a constant value, and with a different set of values for the burstlength parameters

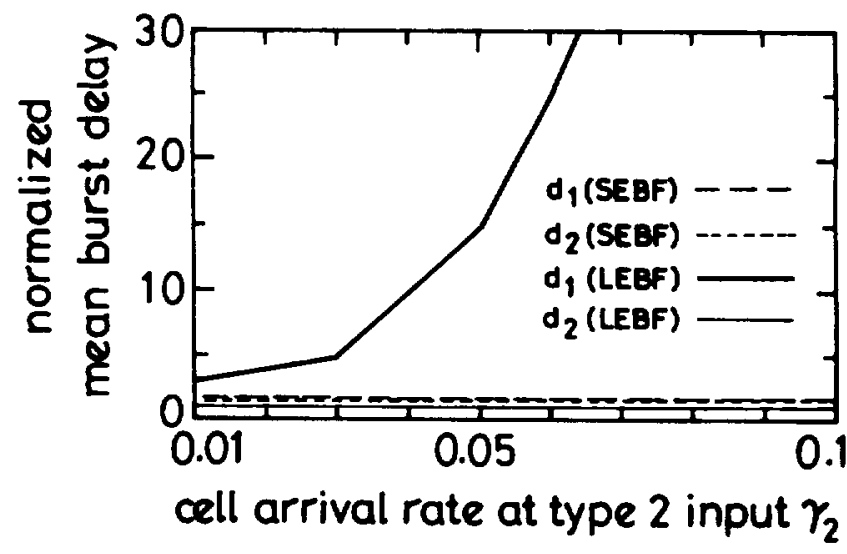

Fig. 8. Normalized mean burst delay for type 1 and type 2 traffic with the two priority selection contention resolution policies; simulation results with $N=16, p_{1}=0.1, p_{2}=0.9, \gamma_{1}=0.6, \alpha=0.5$.

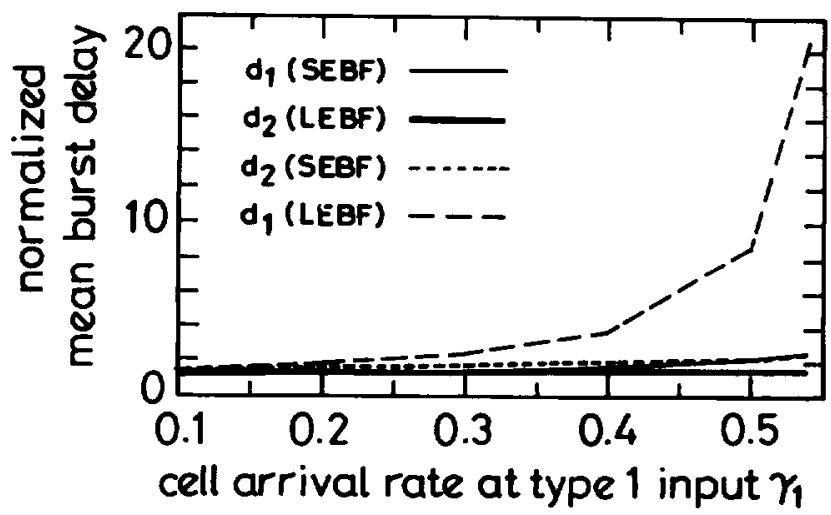

Fig. 9. Normalized mean burst delay for type 1 and type 2 traffic with the two priority selection contention resolution policies; analytical results $(N \rightarrow \infty)$ with $p_{1}=0.3, p_{2}=0.7, \gamma_{2}=0.2, a=0.5$.

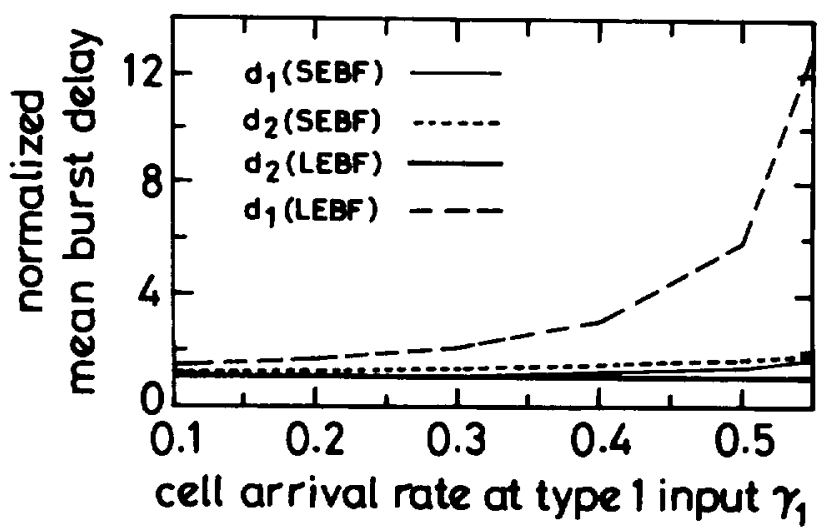

Fig. 10. Normalized mean burst delay for type 1 and type 2 traffic with the two priority selection contention resolution policies; simulation results with $N=16, p_{1}=0.3, p_{2}=0.7, \gamma_{2}=0.2, \alpha=0.5$.

( $p_{1}=0.3$ and $p_{2}=0.7$ ). In all the simulation studies, the cell arrival process described in the Introduction was used.

Figs. 7-12 illustrate some interesting results. There are combinations of cell arrival rates for the two bursty classes such that while both type 1 and type 2 input queues are stable with SEBF policy, type 1 (shorter mean burst length) queues are unstable with reversed priority; further, the degradation 
TABLE III

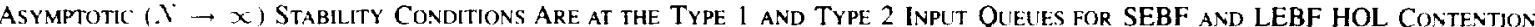
Resolition Strategles, for the Parameters in the Examples in Section IV. il and 12 Are Type 1 and Type 2 Ceil. Arrival Rates; the dashes in the 11 and 12 Columns Mean That Cell Arrival Rates Are ARbitrary: $\overline{0}:=(1-n)$

\begin{tabular}{|c|c|c|c|c|c|c|c|c|}
\hline \multirow[b]{3}{*}{$p_{1}$} & \multirow[b]{3}{*}{$p_{2}$} & \multirow[b]{3}{*}{$\alpha$} & \multirow[b]{3}{*}{$\gamma_{1}$} & \multirow[b]{3}{*}{$\boldsymbol{\gamma}_{2}$} & \multicolumn{4}{|c|}{ asymptotic stability condition } \\
\hline & & & & & \multicolumn{2}{|c|}{$\begin{array}{c}\text { SEBF } \\
\text { (priority to type 1) }\end{array}$} & \multicolumn{2}{|c|}{$\begin{array}{c}\text { LEBF } \\
\text { (priority to type 2) }\end{array}$} \\
\hline & & & & & $\begin{array}{l}\text { type } 1 \text { queues } \\
\alpha^{-1} T_{H}\left(p_{1}, \alpha\right)\end{array}$ & $\begin{array}{c}\text { type 2 queues } \\
\bar{\alpha}^{-1} T_{L}\left(p_{1}, p_{2}, \alpha, \alpha \gamma_{1}\right)\end{array}$ & $\begin{array}{c}\text { type } 1 \text { queues } \\
\alpha^{-1} T_{L}\left(p_{2}, p_{1}, \bar{\alpha}, \bar{\alpha} \gamma_{2}\right)\end{array}$ & $\begin{array}{l}\text { type } 2 \text { queues } \\
\bar{\alpha}^{-1} T_{H}\left(p_{2}, \bar{\alpha}\right)\end{array}$ \\
\hline 0.1 & 0.9 & 0.5 & 0.6 & - & $\gamma_{1}<0.7514$ & $\gamma_{2}<0.4668$ & $r_{2}<0.0601$ & $\gamma_{2}<0.6742$ \\
\hline 0.9 & 0.99 & $0 . \overline{5}$ & 0.6 & - & $\gamma_{1}<0.6742$ & $\gamma_{2}<0.4552$ & $\gamma_{2}<0.0310$ & $\gamma_{2}<0.6674$ \\
\hline 0.3 & 0.7 & 0.5 & - & 0.2 & $\gamma_{1}<0.7286$ & $\gamma_{2}<0.4036$ & $\gamma_{1}<0.5674$ & $\gamma_{2}<0.6906$ \\
\hline
\end{tabular}

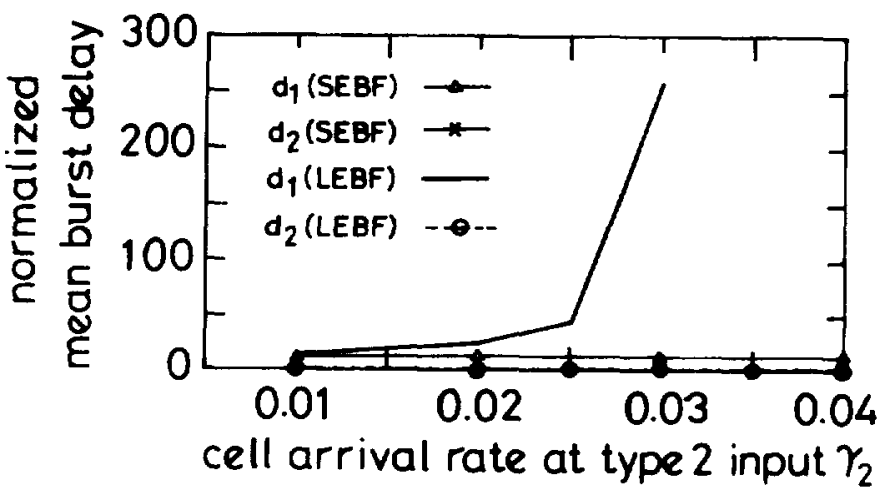

Fig. 11. Normaliesd mean burst delay for type 1 and type 2 traffic with the two priority selection contention resolution policies; analytical results $(.1 \rightarrow x)$ with $y=0.9 . \mu .2=0.99 .11=0.6 . n=0.5$.

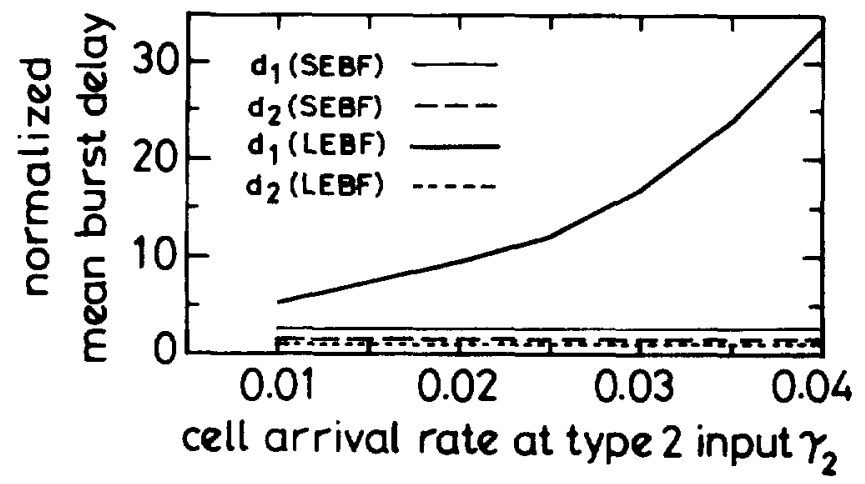

Fig. 12. Normalized mean burst delay for type 1 and type 2 traffic with the two priority selection contention resolution policies; simulation results with $x=16 . \mu_{1}=0.9 . p_{2}=0.99 . ; 1=0.6 . n=0.5$.

of type 2 (longer mean burst length) mean burst delay with SEBF as compared to LEBF is not significant. Thus without significantly affecting the delay performance of type 2 traffic, that of type 1 traffic is improved drastically.

In Fig. 13, we compare the normalized mean burst delays with the RS and SEBF policies, for type 1 and type 2 input traffic. We see the clear advantage of SEBF over RS for type 1 traffic. Also we observe that the type 2 traffic delay performance is almost the same under SEBF and RS for the typical load range shown

The broad conclusions made above could have been anticipated from the asymptotic saturation throughput formulas derived in [7]. We consider two traffic types with burst parameters $p_{1}$ and $p_{2}$. We define $T_{H}\left(p_{1}, \alpha\right)=$ asymptotic

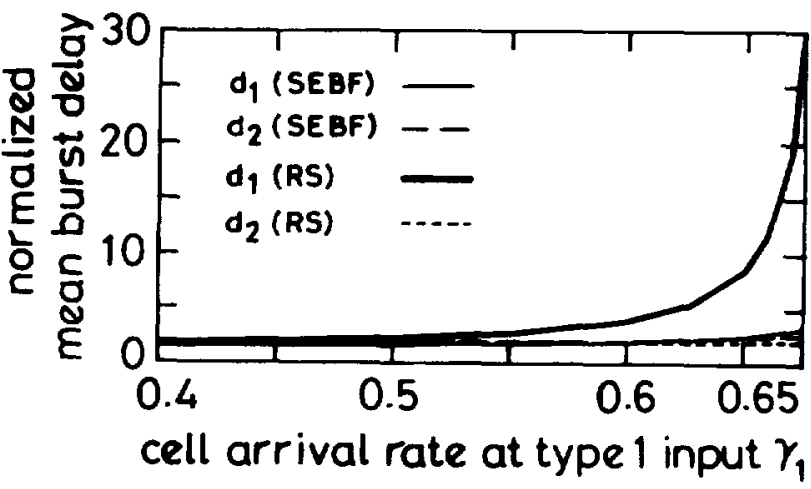

Fig. 13. Normalized mean burst delay for type 1 and type 2 traffic with the random selection and the priority selection contention resolution policies: simulation results with $V=16 . \mu_{1}=0.3 . \mu_{2}=0.7 .2=0.2 .6=0.5$.

(as $N \rightarrow \infty$ ) high priority saturation throughput (at each switch output port) when type 1 traffic occupies a fraction $\alpha$ of the switch inputs, and is given priority on output contention. $T_{L}\left(p_{1}, p_{2}, \alpha . T_{H}\right)=$ asymptotic low priority saturation throughput (at each switch output porf) when type 1 traffic occupies a fraction $\alpha$ of the switch inputs, type 1 traffic is given priority, and the high priority throughput per switch output is $T_{H}$ (of course, $T_{H}<T_{H}\left(p_{1}, \alpha\right)$ ).

The following formulas have been derived in 171 :

$$
\begin{aligned}
& T_{H}\left(p_{1}, \alpha\right) \\
&= \frac{(1+\alpha)-\sqrt{\left\{(1+\alpha)^{2}-2 \alpha\left(1-p_{1}\right)\right\}}}{\left(1-p_{1}\right)} \\
& T_{L}\left(p_{1}, p_{2}, \alpha, T_{H}\right) \\
&= {\left[b\left(p_{1}, p_{2}, \alpha, T_{H}\right)\right.} \\
&\left.-\sqrt{b^{2}\left(p_{1}, p_{2}, \alpha, T_{H}\right)-4 a\left(p_{1}, p_{2}, T_{H}\right) r\left(p_{1}, \alpha, T_{H}\right)}\right] / \\
& {\left[2 a\left(p_{1}, p_{2}, T_{H}\right)\right] }
\end{aligned}
$$

where

$$
\begin{aligned}
a\left(p_{1}, p_{2}, T_{H}\right)= & \left(1-p_{1}\right)\left(1-p_{2}\right)\left(1-T_{H}\right) . \\
b\left(p_{1}, p_{2}, \alpha, T_{H}\right)= & 2(2-\alpha)\left(1-p_{1}\right)\left(1-T_{H}\right) \\
& +\left(1-p_{2}\right)\left[2 T_{H}-T_{H}^{2}\left(1-p_{1}\right)\right] \\
& -2\left(1-p_{1}\right)\left(1-p_{2}\right) T_{H}\left(1-T_{H}\right) . \\
c\left(p_{1}, \alpha, T_{H}\right)= & 2(1-\alpha)\left(1-p_{1}\right)\left(1-T_{H}\right)^{2} .
\end{aligned}
$$

Using these formulas with the parameters in the figures in this section, we get the results shown in Table III. Recall that $\gamma_{i}$ is the cell arrival rate at the type $i$ input; and if the input 
queues are stable with this $\left(\gamma_{1}, \gamma_{2}\right)$ then the throughput at each output is going to be $\left(\alpha \gamma_{1},(1-\alpha) \gamma_{2}\right)$.

In Table III, the first row corresponds to Figs. 7 and 8, the second row corresponds to Figs. 11 and 12, and the third row corresponds to Figs. 9 and 10. All the arrival rates shown in Table III are input queue arrival rates. Observe from the first two rows that with SEBF and $\gamma_{1}=0.6$ the type 1 input queues are stable, since $0.6<0.7514$ and $0.6<0.6742$, while $\gamma_{2}$ can go up to 0.4668 or 0.4552 , respectively. With LEBF, however, while the stable arrival rate for type 2 increases to 0.6742 or 0.6674 (last columns of the first two rows), the type 1 queues (which have a fixed arrival rate of $\gamma_{1}=0.6$ ) become unstable for $\gamma_{2}$ as small as 0.0601 or 0.0301 , respectively. Similarly, in the third row of Table III, with SEBF the type 1 stability condition is $\gamma_{1}<0.7286$. The value in the 7 th column of the third row is $0.5^{-1} T_{L}(0.3,0.7,0.5,0.5 \times 0.7286)=0.4036$, which is the maximum stable arrival rate for type 2 cells when the type 1 queues are saturated; since $\gamma_{2}=0.2<0.4036$, the type 2 queues will be stable since $\gamma_{1}$ must be less than 0.7286. With LEBF, however, while the type 2 stable arrival rate increases to 0.6906 , even with $\gamma_{2}$ as small as 0.2 the type 1 stable arrival rate drops to 0.5674 (from 0.7286 for SEBF).

We can make the following general remarks, from the preceding figures and Table III:

1) Table III corroborates the delays curves in the figures in this section.

2) As observed before, asymptotic saturation throughput is an underestimate for finite-sized switches, but the qualitative comparative conclusions drawn from the asymptotic analysis also apply to switches of finite size.

3) While the saturation throughput results suggest the phenomenon that the delays curves show, the dramatic degradation in type 1 delay with LEBF, and the very slight degradation in type 2 delay with SEBF, can best be appreciated from the delay curves. Hence the delay analysis in this paper is a necessary complement to the saturation analysis in [7].

\section{CONCLUSION}

In this paper, we have studied the impact of cell scheduling strategies on the mean burst delay for a local access ATM switch with mixed bursty traffic. We have found that, unlike Bernoulli arrivals, with bursty cell arrivals, asymptotic (as $N \rightarrow \infty$ ) analysis yields good approximation only for large switch sizes. We have demonstrated a hybrid technique, for estimating input queuing delays, that utilizes an analytically derived effective service time distribution at an input queue, along with simulation of an input queue. We have found, in addition, that the qualitative nature of results predicted by the asymptotic analysis applies well to finite switch sizes.

We showed that the SEBF policy can yield significantly lower mean delay for the short burst length traffic without much effect on the longer burst length traffic. This means that if the less bursty traffic is delay sensitive, then SEBF policy has great advantage. On the other hand, if the more bursty traffic is delay sensitive then by giving it a low priority (as in SEBF), we may degrade the delay performance of this traffic but not significantly. Recall from the Introduction that a motivating scenario for our model was that the less bursty traffic could be from metropolitan or campus area networks. Such traffic would carry interactive services, e.g., interactive simulations with an image on a graphics workstation in one location being constantly updated by a program running on a supercomputer in a different location. Such traffic would have short bursts but would be delay sensitive. We also find that the delay performance of RS lies in between that of the two static priority policies; hence RS can be an adequate compromise if there is no prior knowledge of input traffic burstiness.

\section{APPENDIX \\ MEan Low PRiority HOL Contention Delay}

At the end of a slot in which a low priority cell is served, a high priority busy period (possibly of zero slot duration) is initiated by a Poisson batch of high priority bursts. At the termination of the busy period again a low priority cell gets service. We call the high priority busy period followed by the one slot service time of a low priority cell, together as a low priority cell completion time.

Define the following random variables

1) $C_{1}$ (respectively $C_{2}$ ) := Poisson batch size of high priority (resp. low priority) HOL bursts that arrive at the beginning of each time slot,

2) $B:=$ high priority busy period initiated by a Poisson batch of high priority HOL bursts (this "busy period" could be of zero slot duration if the Poisson batch is empty),

3) $G:=$ low priority cell completion time $(=B+1)$,

4) $\Gamma:=$ total number of low priority bursts arriving at the HOL contention process during one low priority cell completion time,

5) $F:=$ total service time (in no. of slots) of a Poisson batch of high priority bursts.

Then with the usual notation for the pgfs and pmfs (see Section I-A), and recalling that the high priority burst length is geometric with parameter $p_{1}$, we obtain the following expressions:

$$
\begin{aligned}
\tilde{f}(z) & =\sum_{k=0}^{\infty}\left[\frac{z\left(1-p_{1}\right)}{1-z p_{1}}\right]^{k} \frac{e^{-\alpha \lambda_{1}}\left(\alpha \lambda_{1}\right)^{k}}{k !} \\
& =e^{-\alpha \lambda_{1}(1-z) /\left(1-z p_{1}\right)} .
\end{aligned}
$$

Observe that $F$ can be viewed as the total amount of work (in slots) brought in by an arriving batch of high priority HOL bursts. For a work-conserving server, the busy period distribution is invariant to the service discipline. Hence, to compute the busy period distribution, we consider the following discipline: serve one cell of the first arriving batch, and then serve the busy period of the Poisson batch of bursts that arrives after this slot; the total time for this will have pgf $z \tilde{b}(z)$. This is repeated $k$ times with probability $f(k)$. Hence

$$
\begin{aligned}
\tilde{b}(z) & =\sum_{k=0}^{\infty}[z \tilde{b}(z)]^{k} f(k) \\
& =\tilde{f}(z \tilde{b}(z)) .
\end{aligned}
$$


Further, since $C=B+1$

$$
\dot{g}(z)=z \dot{f}(z \dot{b}(z))
$$

and, noting that $C \geq 1$

$$
\begin{aligned}
\dot{\gamma}(z) & =\sum_{n=1}^{\infty}\left[\bar{c}_{2}(z)\right]^{n} g(n) \\
& =\tilde{g}\left[\hat{c}_{2}(z)\right]
\end{aligned}
$$

where $i_{2}(z)$ is the pgf of the low priority arriving batch.

Recall that we are focusing on the HOL contention process which a tagged low priority burst has joined. Consider the ends of those slots in which a low priority cell is transmitted. Let $\left\{X_{k}^{-}, k \geq 1\right\}$ denote the number of low priority HOL bursts at the kth such epoch, where $X_{k}^{-}$does not include the newly arriving low priority HOL bursts, nor does it include the burst corresponding to the cell just transmitted when this burst is not yet complete (i.e., this burst is viewed as a feedback arrival). Owing to the asymptotic analysis and the consequent i.i.d. arrivals of new HOL bursts of both priorities, $\left\{X_{k}^{-}, k \geq 1\right\}$ is a Markov chain.

$$
X_{k+1}^{-}=X_{k}^{-}+A_{k+1}-1
$$

where $X_{k}^{-}+A_{k+1} \geq 1$ since we consider the ends of those slots in which a low priority cell is transmitted. In the above expression

$$
A_{k+1}=A_{k+1}^{-}+Z_{k} .
$$

where $Z_{k}$ ( $=0$ if the burst just served is complete, and $=1$ otherwise) is a sequence of i.i.d random variables with

$$
Z_{k}= \begin{cases}1 & \text { w.p. } p_{2} \\ 0 & \text { w.p. }\left(1-p_{2}\right) \triangleq \bar{p}_{2}\end{cases}
$$

and $A_{k+1}^{-}$is the number of low priority burst arrivals after the end of the $k$ th low priority service completion epoch, but before the end of the $(k+1)$ st such epoch. It is easily seen that

$$
P\left(A_{k+1}=i\right)=\gamma(i) . \quad i \geq 0 \text { if } X_{k}^{-}+Z_{k} \geq 1
$$

and further, if $X_{k}^{-}+Z_{k}=0$, then a low priority cell is there to be served at the end of a high priority busy period in which at least one low priority burst arrives; hence

$$
P\left(A_{k+1}^{-}=i\right)=\frac{\gamma(i)}{1-\gamma(0)} . \quad i \geq 1 \quad \text { if } \quad X_{k}^{-}+Z_{k}=0
$$

$\left\{\mathrm{X}_{k}^{-}, k \geq 1\right\}$ is an embedded Markov chain and its transition probability matrix has the form

\begin{tabular}{c|cccccccc} 
& 0 & 1 &. & $i-1$ & $i$ & $i+1$ &. &. \\
\hline 0 & $w(0)$ & $w(1)$ & &. & $w(i)$ &. &. &. \\
1 & $t(0)$ & $t(1)$ &. & $t(i)$ &. &. &. \\
2 & 0 & $t(0)$ & &. & $t(i-1)$ &. &. &. \\
. &. &. &. &. &. &. &. &. \\
. &. &. &. &. &. &. &. &. \\
$i$ & 0 &. &. & $t(0)$ & $t(1)$ & $t(2)$ & $t(3)$ &. \\
. &. &. &. &. &. &. &. &. \\
. &. &. &. &. &. &. &. &.
\end{tabular}

where

$$
\begin{array}{rlrl}
\forall j \geq 0 . & u(j) & =\gamma(j) p_{2}+\frac{\gamma(j+1) \bar{p}_{2}}{1-\gamma(0)} \\
t(0) & =\gamma(0) \bar{p}_{2} \\
\forall j \geq 1, & t(j)=\gamma(j-1) p_{2}+\gamma(j) \bar{p}_{2}
\end{array}
$$

It follows that the equilibrium distribution $\left\{r^{-}(j), j \geq 0\right\}$ is given by

$$
x^{-}(j)=x^{-}(0) w(j)+\sum_{k=1}^{j+1} x^{-}(k) t(j+1-k) .
$$

Taking transform and rearranging terms

$$
\tilde{x}^{-}(z)=\frac{r^{-}(0)[\tilde{t}(z)-z \tilde{u}(z)]}{[\tilde{t}(z)-z]}
$$

where

$$
\begin{aligned}
\tilde{w}(z) & =\sum_{j=0}^{\infty} z^{j}\left[p_{2} \gamma(j)+\bar{p}_{2} \frac{\gamma(j+1)}{1-\gamma(0)}\right] \\
& =p_{2} \bar{\gamma}(z)+\bar{p}_{2} \frac{\tilde{\gamma}(z)-\gamma(0)}{z[1-\gamma(0)]} \\
\tilde{t}(z) & =\gamma(0) \bar{p}_{2}+\sum_{j=1}^{\infty}\left[p_{2} \gamma(j-1)+\bar{p}_{2} \gamma(j)\right] z^{j} \\
& =\tilde{\gamma}(z)\left(\bar{p}_{2}+z p_{2}\right) .
\end{aligned}
$$

Substituting (A.3) and (A.4) in (A.2) and simplifying, we obtain

$$
\begin{aligned}
& \tilde{x}^{-}(z)=\frac{x^{-}(0) \bar{p}_{2} \gamma(0)[1-\dot{\gamma}(z)]}{[1-\gamma(0)]\left[\dot{\gamma}(z)\left(\bar{p}_{2}+z p_{2}\right)-z\right]} \\
& \dot{r}^{-}(1)=\frac{x^{-}(0) \gamma(0) E \Gamma \bar{p}_{2}}{[1-\gamma(0)]\left(1-p_{2}-E \Gamma\right)}
\end{aligned}
$$

also

$$
\tilde{x}^{-}(1)=1 \text {. }
$$

It follows that

and

$$
x^{-}(0)=\frac{\left(1-p_{2}-E \Gamma\right)[1-\gamma(0)]}{\bar{p}_{2} \gamma(0) E \Gamma}
$$

$$
\tilde{x}^{-}(z)=\frac{\left(1-p_{2}-E \Gamma\right)[1-\dot{\gamma}(z)]}{E \Gamma\left[\dot{\gamma}(z)\left(1-p_{2}+z p_{2}\right)-z\right]}
$$

where $E \Gamma$ is the expectation of $\Gamma$, and is obtained from the pgf of $\Gamma$ (A.1).

Continuing the analysis, let $k$ index the successive slots. Let $\left\{X_{k}, k \geq 1\right\}$ denote the number of low priority bursts, in the tagged HOL contention process, at the end of the $k$ th slot, after the possible low priority burst departure in that slot but before new HOL burst arrivals (see Fig. 14). Let $\left\{\sigma_{i}, i \geq 1\right\}$ denote the slot indices in which a low priority cell is served in the tagged HOL contention process. From the definition of the process $\left\{X_{k}^{-}\right\}$above, it is then clear that, for $i \geq 1$

$$
X_{\sigma_{i}}=X_{i}^{-}+Z_{i}
$$

with $Z_{i}$ as defined above. Let $\left\{\sigma_{i}, j \geq 1\right\}$ be the subsequence of $\left\{\sigma_{i}, i \geq 1\right\}$ such that $Z_{i,}=0 . j \geq 1$, i.e., $X_{\sigma_{1}}=X_{i}^{-}$. 


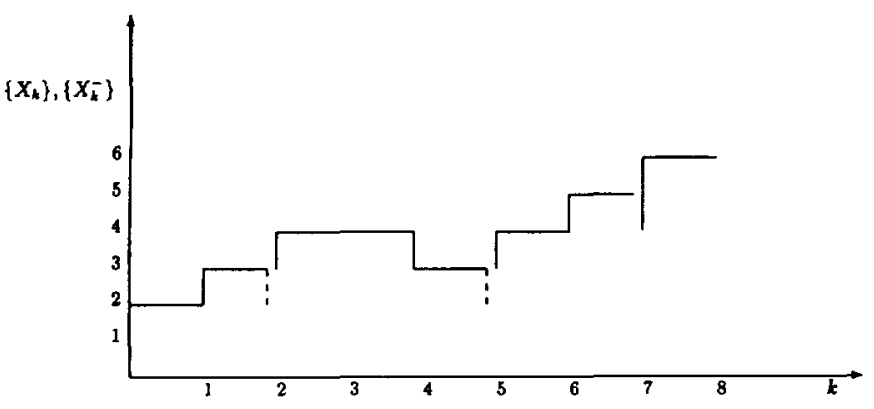

Fig. 14. Pictorial depiction of the processes $\left\{X_{k}\right\}$ and $\left.\left\{X_{k}^{-}\right\}\right]$. Here $x_{1}=2, x_{2}=3, x_{3}=4, x_{4}=3, x_{5}=3, x_{6}=4, x_{7}=4$. Cell services occur in slots $2,4,5$, and 7. Hence, $X_{1}^{-}=2, X_{2}^{-}=3, X_{3}^{-}=2$, $\mathrm{X}_{4}^{-}=4$. The solid lines show the transitions in $\left\{X_{k}\right\}$. The dashed lines show the "pseudo" transitions in which a serviced cell is immediately fed back.

$j \geq 1$. The epochs $\left\{\sigma_{i_{j}}, j \geq 1\right\}$ are low priority burst departure epochs. Since $\left\{Z_{i}, i \geq 1\right\}$ is a Bernoulli process, it follows that the subsequence $\left\{X_{i_{j}}^{-}, j \geq 1\right\}$ of the process $\left\{X_{i}^{-}, i \geq 1\right\}$ is obtained by Bernoulli sampling of $\left\{X_{i}^{-}, i \geq\right.$ $1\}$.

For $m \geq 0$, define the following stationary probabilities for the process $\left\{X_{k}, k \geq 1\right\}$ :

1) $x(m): \operatorname{Pr}\left\{X_{k}=m\right\}$,

2) $d(m): \operatorname{Pr}\{$ a low priority burst departure leaves behind $m$ low priority bursts $\}$, and

3) $a(m): \operatorname{Pr}$ \{an arriving nonempty batch of low priority bursts finds $m$ low priority bursts already in the HOL process .

Note that since the low priority burst arrivals are i.i.d, the epochs of arrivals of nonempty bursts is a Bernoulli sequence. From the preceding observations it follows, using the discrete time version of PASTA, i.e., GASTA [4] that

$$
\begin{aligned}
& d(m)=x^{-}(m) \quad m \geq 0 \\
& a(m)=x(m) \quad m \geq 0 \text {. }
\end{aligned}
$$

We now use a level crossing argument on $\left\{X_{k}, k \geq 1\right\}$ to relate $d(m)$ and $a(m)$, and hence to relate $x(m)$ and $x^{-}(m)$. Let, for $m \geq 0, n \geq 1$,

1) $\operatorname{up}_{n}(m)$ : number of up-transitions in $\left\{X_{k}, 1 \leq k \leq n\right\}$ that cross level $m$, and

2) down $n_{n}(m)$ : number of down-transitions $(m+1) \downarrow m$ in $\left\{X_{k}, 1 \leq k \leq n\right\}$.

Clearly

$$
\left|\operatorname{up}_{n}(m)-\operatorname{down}_{n}(m)\right| \leq 1
$$

It follows that

$$
\lim _{n \rightarrow \infty} \frac{\operatorname{up}_{n}(m)}{n}=\lim _{n \rightarrow \infty} \frac{\operatorname{down}_{n}(m)}{n}
$$

Let

$$
\delta_{n}=\text { no. of burst departures in }[0, n]
$$

then

$$
\lim _{n \rightarrow \infty} \frac{\operatorname{down}_{n}(m)}{n}=\lim _{n \rightarrow \infty} \frac{\delta_{n}}{n} \frac{\operatorname{down}_{n}(m)}{\delta_{n}}
$$

Now, for a stable system

$$
\lim _{n \rightarrow \infty} \frac{\delta_{n}}{n}=E C_{2} \quad \text { w.p. } 1
$$

(where w.p. 1 denotes "with probability one") and

$$
\lim _{n \rightarrow \infty} \frac{\operatorname{down}_{n}(m)}{\delta_{n}}=d(m) \quad \text { w.p. } 1 \text {. }
$$

Further, defining $\bar{C}_{2}(j)=\sum_{i=j}^{\infty} c_{2}(i)$

$$
\begin{aligned}
& \lim _{n \rightarrow \infty} \frac{\operatorname{up}_{n}(m)}{n}=\left[1-c_{2}(0)\right] \\
& \cdot\left[\frac{a(0) \bar{C}_{2}(m+1)}{1-c_{2}(0)}+\frac{a(1) \bar{C}_{2}(m)}{1-c_{2}(0)}\right. \\
&\left.+\cdots+\frac{a(m) \bar{C}_{2}(1)}{1-c_{2}(0)}\right] \text { w.p. } 1
\end{aligned}
$$

thus, w.p. $1, \forall m \geq 0$

$$
E C_{2} d(m)=\sum_{t=0}^{m} a(i) \bar{C}_{2}(m+1-i)
$$

Taking transforms

$$
\begin{aligned}
E C_{2} \tilde{d}(z) & =\sum_{m=0}^{\infty} z^{m} \sum_{i=0}^{m} a(i) \bar{C}_{2}(m+1-i) \\
& =\sum_{i=0}^{\infty} a(i) \sum_{m=i}^{\infty} \bar{C}_{2}(m+1-i) z^{m+1-i} z^{i-1} \\
& =\left[\tilde{\bar{C}}_{2}(z)-1\right] z^{-1} \tilde{a}(z)
\end{aligned}
$$

or

$$
\tilde{a}(z)=\frac{z E C_{2} \tilde{d}(z)}{\left[\tilde{\bar{C}}_{2}(z)-1\right]} .
$$

It follows from (A.6) and (A.7) that

$$
\tilde{x}(z)=\frac{z E C_{2} \tilde{x}^{-}(z)}{\left[\tilde{\bar{C}}_{2}(z)-1\right]}
$$

where

$$
\begin{aligned}
\tilde{\bar{C}}_{2}(z) & =\sum_{k=0}^{\infty} z^{k} \sum_{j=k}^{\infty} c_{2}(j) \\
& =\frac{1-z \tilde{c}_{2}(z)}{1-z}
\end{aligned} .
$$

Substituting (A.5) in (A.8) and simplifying, we get

$$
\tilde{x}(z)=\frac{E C_{2}\left(1-p_{2}-E \Gamma\right)(1-z)[1-\tilde{\gamma}(z)]}{E \Gamma\left[1-\tilde{c}_{2}(z)\right]\left[\tilde{\gamma}(z)\left(\bar{p}_{2}+p_{2} z\right)-z\right]} .
$$

By applying Little's Law, we obtain the mean sojourn time $S_{L}$ of the low priority burst in the HOL process, i.e., the mean HOL contention time of a low priority burst. Observe, however, from the way $\left\{X_{k}, k \geq 1\right\}$ is embedded in the HOL 
contention process, that a burst that sojourns for $m$ slots in the process, contributes to $\left\{X_{k}\right\}$ only for $m-1$ slots. Thus Little's Law yields

$$
\begin{aligned}
\frac{E X}{E C_{2}} & =E\left(S_{L}-1\right) \\
& =E S S_{L}-1
\end{aligned}
$$

where $E X$ is obtained from the pgf of $X$ (A.9).

This result for $E S_{I}$ is perhaps the most complete and iccurate closed form result we can hope to get for this problem. It only involves the (asymptotically accurate) assumption of Poisson arrivals. This result is used to check the accuracy of numerical results to be obtained from the analysis in Section II-B1.

\section{REFERENCES}

[1] W. R. Byme et al. "Evolution of metropolitan area networks to broadband ISDN," IEEE Commun. Mag., vol. 29, no. 1, pp. 69-82, Jan. 1991.

[2] J. S.-C. Chen and R. Guerin, "Performance study of an input queuing packel switch with two priority classes," IEEE Trans. Commun., vol. 39. no. 1. pp. 117-126, Jan. 1991.

13] A. Descloux, "Stochastic models for ATM switching networks," IEEE J. Selectr. Areas Commun.. vol. 9, no. 3, pp. 450-457, Apr. 1991.

[4] S. Halfin, "Batch delays vs. customer delays," AT\&T Bell Labs Tech. Memorandum, 1982.

[5] M. G. Hluchyj and M. J. Karol, "Queuing in high performance packet switching," IEEE J. Select. Areas Commun., vol. 6, no. 9, pp. 1587-1597, Dec. 1988.

[6] J. Y. Hui and E. Arthurs, "A broadband packet switch for integrated transport," IEEE J. Select. Areas Commun., vol. 5, no. 8, pp. 1264-1273, Oct. 1987.

[7] L. Jacob and A. Kumar. "Saturation throughput analysis of an input queuing ATM switch with multiclass bursty traffic" IEEE Trans. Commun., vol. 43, no. 2/3/4, Feb.Mar./Apt. 1995.

18] L. Jacob, "Performance analysis of scheduling strategies for switching and multiplexing of multiclass variable bit rate traffic in an ATM network," Ph.D. dissertation, Indian Institute of Science, Bangalore, Dec. 1992.

[9] D. L. Jagerman. "An inversion technique for the Laplace transform," Bell Syst. Tech. J. vol 61, no. 8, pp. 1995-2002, Oct 1982.

[10] M. J. Karol. M. G. Hluchyj, and S. P. Morgan, "Input versus output queuing on a space-division packet switch," IEEE Trans. Commun., vol. 35. no. 12. pp. 1347-1356. Dec. 1987.

(11) L. Kleinrock, Queuing Systems, vol. 1. New York: John Wiley, 1975

[12] S. Q. Li, "Nonuniform traffic analysis on a nonblocking space-division packet switch," IEEE Trans. Commun., vol. 38, no. 7, pp. 1085-1096, July 1990
[13] _. "Performance of a nonblocking space-division packet switch with correlated input traffic," in Proc. 1989 GLOBECOM Conf.. pp. 1754-1763

[14] S. C. Liew and K. W. Lu, "Comparison of buffering strategies for asymmetric packet switch modules," IEEE J. Seledt. Areas Cummun. vol. 9. no. 3. pp. 428-438, Apr. 1991.

[15] T. Meisling. "Discretetime queuing theory," Oper, Res., vol. 6. pp 96-105, Jan./Feb. 1958.

[16] M. F. Neuts, Matrix-Geometric Solutions in Stochastic Models: An Algorithmic Appmach. Baltimore, MD: The John Hopkinu l nivervity Press, 1981

[17] R. W. Wolff, Siochastic Modeling and the Theory of Queues. Englewood Cliffs, NJ: Prentice-Hall, 1989.

[18] S. Wolfram, Mathematica, A System for Doing Mathematics by Computer. Reading. MA: Addison-Wesley. 1988

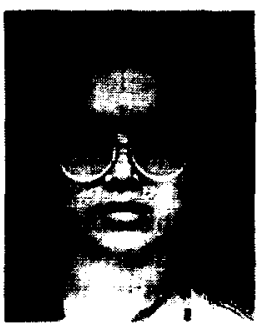

Lillykutty Jacob received the B.Sc. (Eng.) degree in electronics and communication from the Kerala University, India, in 1983, the M.Tech. degree in electrical engineering (communication) from the Indian Institute of Technology, Madras, India, in 1985 , and the Ph.D. degree in electrical communication engineering from the Indian Institute of Science. Bangalore, India. in 1993.

Since 1985, she has been with the Deparment of Electrical Engineering, Regional Engineering College, Calicut, India. Currently, she is an Assistant Professor there. Her research interests include performance modeling and analysis/simulation, personal communication systems, and ATM networks.

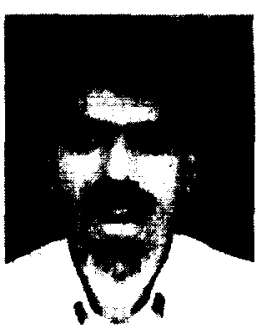

Anurag Kumar (SM'92) received the B.Tech. degree in electrical engineering from the Indian In stitute of Technology at Kanpur in 1977. He then obtained the Ph.D. degree from Comell University in 1981.

He was a Member of Technical Staff at AT\&T Bell Labs, Holmdel, NJ, for more than six years. During this period, he worked on the performance analysis of computer systems, communication networks, and manufacturing systems. Since 1988, he has been with the Indian Institute of Science (IISc). Bangalore, in the Dept. of Electrical Communication Engineering. where he is now Associate Professor. He is also the Coordinator at IISc of the Education and Research Network Project, which has set up a country-wide computer network for academic and research institutions, and conducts $R \& D$ and training in the area of communication networks. His own research and consultancy interests are in the area of modeling, analysis, control, and optimization problems arising in communication networks and distributed systems.

Dr. Kumar was awarded the President of India's Gold Medal in 1977. 